\title{
Fossil record of holococcoliths and selected hetero-holococcolith associations from the Mediterranean (Holocene-late Pleistocene): Evaluation of carbonate diagenesis and palaeoecological-palaeocenographic implications
}

\author{
Daniela Crudeli ${ }^{\mathrm{a}, *}$, Jeremy R. Young ${ }^{\mathrm{b}}$, Elisabetta Erba ${ }^{\mathrm{a}}$, Markus Geisen ${ }^{\mathrm{c}}$, \\ Patrizia Ziveri ${ }^{\mathrm{d}}$, Gert J. de Lange ${ }^{\mathrm{e}}$, Caroline P. Slomp ${ }^{\mathrm{e}}$ \\ a Department of Earth Sciences "Ardito Desio", University of Milan, Via Mangiagalli 34, 20133 Milan, Italy \\ b Palaeontology Department, Cromwell Road, The Natural History Museum, London SW7 5BD, UK \\ c Alfred Wegener Institute for Polar and Marine Research, Am Handelshafen 12 D-27570 Bremerhaven, Germany \\ ${ }^{\mathrm{d}}$ Department of Paleoecology and Paleoclimatology, Faculty of Earth and Life Sciences, De Boelelaan 1085, \\ 1081HV Amsterdam, The Netherlands \\ ${ }^{\mathrm{e}}$ Department of Earth Sciences - Geochemistry, Faculty of Geosciences, Budapestlaan 4, 3584 CD Utrecht, The Netherlands
}

Received 19 January 2005; received in revised form 18 November 2005; accepted 28 November 2005

\begin{abstract}
The Holocene-late Pleistocene distribution of holococcoliths, is quantified by light microscopy from cores from the Western Mediterranean, the Aegean Sea and eight eastern Mediterranean cores recovering sapropel S1. The diversity of fossil holococcoliths is much lower than is seen in the plankton, indicating selective preservation. However the holococcolith phases of Syracosphaera pulchra and Helicosphaera carteri are abundantly preserved allowing a comparison of fossil records of heterococcolith and holococcolith phases of these species.

In shallow cores a primary palaeocological signal appears to be preserved, suggesting that under suitable circumstances it is possible to use holococcoliths in palaeoceanography. The common occurrence of holococcoliths in the sapropels from these cores suggests low surface-water productivity, supporting previous inferences that productivity enhancement during sapropel deposition was confined to the deep-chlorophyll maximum.

In deeper cores there is dramatic decrease in abundance of $S$. pulchra holococcoliths within sapropel sediments. From comparison of shallow and deep cores and from Emiliania huxleyi preservation data we conclude that this is a preservation signal. Evidently the calyptroliths produced by $S$. pulchra have a lower preservation potential than the syracoliths produced by $H$. carteri, we interpret this as a product of their more open structure.
\end{abstract}

(C) 2005 Elsevier B.V. All rights reserved.

Keywords: Holococcoliths; Life-cycles; Sapropel S1; Carbonate diagenesis; Palaeoecology; Palaeoceanography

* Corresponding author. Present address: Department of Geological Sciences and Geotechnologies, University of Milano-Bicocca, Piazza della Scienza 4, 20126 Milan, Italy. Tel.: +39 02 64484334; fax: +39 026448273 .

E-mail address: crudelidaniela@tiscali.it (D. Crudeli).

\section{Introduction}

Coccolithophores are a significant group of pelagic unicellular algae producing and bearing minute calcium 
carbonate platelets-coccoliths. These constitute an important component of deep-sea sediments and are invaluable for reconstruction of past environmental-oceanographic changes. Recent research on coccolithophore biology (Thierstein and Young, 2004) has highlighted the distinctive nature of their life-cycles, and the importance of this in understanding their ecology. In the fossil record there is the possibility that we can apply these new insights by comparing the distribution of holococcoliths and heterococcoliths, here we attempt a first study of this type through detailed examination of assemblages from the Holocene of the Mediterranean.

Two major types of coccoliths can be distinguished: Heterococcoliths, which are formed of a radial array of interlocked crystal-units of variable architecture and size, and holococcoliths, which are formed of numerous minute (ca. $0.1 \mu \mathrm{m}$ ), identical, non-interlocked, euhedral crystallites (e.g., Young et al., 1999). Usually, only one of these types of coccoliths occurs on a single coccosphere, and formerly heterococcoliths and holococcoliths were considered as defining separate families (e.g., Perch-Nielsen, 1985). There was, however, one well-documented case of a coccolithophore, $\mathrm{Coc}$ colithus pelagicus, producing holococcoliths and heterococcoliths in alternate life-cycle phases (Parke and Adams, 1960; Rowson et al., 1986), and suspicion that this might be a common pattern (e.g., Billard, 1994). Recently, culture studies of life-cycle changes (Fresnel, 1994; Houdan et al., 2004; Noël et al., 2004) and observations from water samples of rare combination coccospheres involving both coccolith (e.g., Cros et al., 2000; Geisen et al., 2002), have demonstrated that haplo-diplontic life-cycles are common in coccolithophores. Heterococcoliths are produced in the diploid life-cycle phase and holococcoliths in the haploid phase, with both phases being capable of asexual reproduction.

Cros et al. (2000) observed depth separation between the life-cycle phases of a few species and suggested, that heteromorphy represents an ecological strategy of coccolithophores to survive under different environmental conditions. Similarly, Noël et al. (2004) showed a relationship between changing culture conditions and phase changes in Calyptrosphaera sphaeroidea. In both cases the diploid, heterococcolith-bearing phase appeared to be adapted to more eutrophic conditions and the haploid, holococcolith-bearing phase to more oligotrophic conditions. There is, however, still an imperfect knowledge of the significance of the life cycle. Unfortunately, the fossil record of holococcolithophores is limited due to their low preservation potential (Schneidermann, 1977; Tappan, 1980; Stein- metz, 1991; Ziveri et al., 2000; Young et al., 2005) so palaeontological observations cannot usually be used to address this problem.

The Mediterranean is characterised by a highly diverse holococcolithophore community (Kleijne, 1991; Cros et al., 2000; Cros and Fortuño, 2002; Triantaphyllou and Dimiza, 2003). In addition, the Mediterranean water is saturated in calcite resulting in enhanced preservation of holococcoliths in the sediments. However, few studies have looked at their Late Quaternary fossil record because of the difficulty of identifying of such small forms by light microscopy (Crudeli and Young, 2003, and references therein).

Crudeli and Young (2003) combined scanning electron microscope (SEM) and light microscope (LM) observations to develop a robust LM taxonomy of holococcoliths preserved in the Holocene-late Pleistocene fossil record of the eastern Mediterranean and carried out some preliminary studies of their distribution.

In this work, we document the fossil record (Holocene-late Pleistocene) of holococcoliths and compare the heterococcolith record of Helicosphaera carteri and Syracosphaera pulchra with the associated holococcolith life-cycle phases (Young et al., 2003, and references therein). In particular, distribution changes associated with sapropel S1 are studied. The goal is to assess and interpret the temporal and spatial distribution of holococcoliths and past biodiversity in the Mediterranean basin and to improve our understanding of coccolithophore life cycles.

\subsection{Sapropel S1}

The normal Holocene sedimentation of the eastern Mediterranean was interrupted between $\sim 9.5$ and 6.0 to $5.3 \mathrm{kyr}$ BP (Mercone et al., 2000) by the deposition of a distinctive dark organic carbon-rich level, the S1 sapropel (Cita et al., 1977). This is the youngest of the cyclic organic-carbon rich intervals, which have formed in the basin since the Miocene. They are the product of environmental changes induced by amplification of the African summer monsoon at times of the precession minimum (Rossignol-Strick et al., 1982; Rossignol-Strick, 1985; Lourens et al., 1996). Enhanced freshwater input from the basin borderland induces stratification of the water column, causing decreased bottom water oxygenation. Increased primary production, decreased bottom water ventilation or the combined effects of processes favoured preservation of organic matter at the sea floor (Rohling, 1994; de Lange et al., 1999; Emeis et al., 2000, for synthesis). 
Table 1

Details of the studied cores

\begin{tabular}{|c|c|c|c|c|c|c|c|}
\hline Core name & Core type & Cruise & Position & $\begin{array}{l}\text { Depth } \\
\text { (m) }\end{array}$ & $\begin{array}{l}\text { Length } \\
(\mathrm{cm})\end{array}$ & $\begin{array}{l}\text { No. samples } \\
\text { analysed }\end{array}$ & $\begin{array}{l}\text { Resolution } \\
(\mathrm{cm})\end{array}$ \\
\hline MT15, sec. 10-9 & GC & Tyro 1993 & $38^{\circ} 53.75^{\prime} \mathrm{N}-04^{\circ} 30.60^{\prime} \mathrm{E}$ & 2373 & 141 & 71 & 2 \\
\hline UM42 & $\mathrm{BC}$ & Urania 18, 1994 & $34^{\circ} 57.23^{\prime} \mathrm{N}-17^{\circ} 51.75^{\prime} \mathrm{E}$ & 1375 & 35 & 35 & 1 \\
\hline AP01.1, sec. 1 & $\mathrm{GC}$ & Urania 1998 & $39^{\circ} 12.99^{\prime} \mathrm{N}-19^{\circ} 06.78^{\prime} \mathrm{E}$ & 811 & 80 & 53 & 1 \\
\hline SL114 & $\mathrm{BC}$ & Logachev 1999 & $35^{\circ} 17.24^{\prime} \mathrm{N}-21^{\circ} 24.52^{\prime} \mathrm{E}$ & 3390 & 49.5 & 48 & 1 \\
\hline SL73 & $\mathrm{BC}$ & Logachev 1999 & $39^{\circ} 39.67^{\prime} \mathrm{N}-24^{\circ} 30.65^{\prime} \mathrm{E}$ & 339 & 38.9 & 37 & 1 \\
\hline $\mathrm{BC} 3$ & $\mathrm{BC}$ & Marion Dufresne 81, 1994 & $33^{\circ} 22.51^{\prime} \mathrm{N}-24^{\circ} 46.00^{\prime} \mathrm{E}$ & 2180 & 86 & 59 & 1.2 \\
\hline SL60, sec. $5,6,7$ & $\mathrm{PC}$ & Logachev 1999 & $35^{\circ} 39.69^{\prime} \mathrm{N}-26^{\circ} 34.99^{\prime} \mathrm{E}$ & 1522 & 210 & 103 & 2 \\
\hline BC19 & $\mathrm{BC}$ & Marion Dufresne 69, 1991 & $33^{\circ} 47.85^{\prime} \mathrm{N}-28^{\circ} 36.50^{\prime} \mathrm{E}$ & 2750 & 35 & 34 & 1 \\
\hline SL9 & $\mathrm{BC}$ & Logachev 1999 & $34^{\circ} 17.17^{\prime} \mathrm{N}-31^{\circ} 31.36^{\prime} \mathrm{E}$ & 2302 & 45.2 & 44 & 1 \\
\hline SL29 & $\mathrm{BC}$ & Logachev 1999 & $33^{\circ} 23.35^{\prime} \mathrm{N}-32^{\circ} 30.18^{\prime} \mathrm{E}$ & 1587 & 39.5 & 38 & 1 \\
\hline
\end{tabular}

\subsection{Carbonate diagenesis}

Crudeli et al. (2004) have shown, that Emiliania huxleyi coccoliths are differentially affected by carbonate diagenesis in Holocene-late Pleistocene Mediterranean sediments: Carbonate overgrowth affects coccoliths especially outside S1, whereas this process appears to be weak within the sapropel. Variable diagenesis of coccoliths is probably related to changes in the carbonate system, that also result in an alternation from high-Mg calcite outside the sapropels and low-Mg calcite and aragonite dominated sediments within the sapropels (Calvert and Fontugne, 2001; Thomson et al., 2004). Fragmentation and etching of coccoliths have been documented within the sapropel (Crudeli and Young, 2003; Crudeli et al., 2004).

The comparatively good preservation potential of most heterococcoliths has been assessed by different works (e.g., Roth and Coulbourn, 1982), but almost no information is available on holococcolith preservation. A chemical experiment on the preservation potential of calcareous nannofossils from sapropel and non-sapro- pel sediments was conducted by Castradori (1992) and de Lange et al. (1994). They observed a progressive decrease in concentration of Syracosphaera species 1 (sensu Castradori, 1992), a form absent in the sapropels and variably abundant in the enclosing marls (Negri et al., 1999; Corselli et al., 2002), and only moderate variations in other coccoliths under more corrosive pore water conditions. There is a need to constrain the preservation potential of holococcoliths. We resolve this by coupling SEM observations with considerations on shape-structure of different forms and by comparing the holococcoliths' fossil record to that of E. huxleyi morphotypes.

\section{Materials and methods}

This work is based on four cores from the Ionian Sea, three cores from the Eastern Mediterranean and a core from the Aegean Sea recovering the sapropel S1 and a core from the Cretan Sea containing a rapidly accumulated S1 divided in two units (Table 1; Fig. 1). In addition, a co-eval core from the Western Mediter-

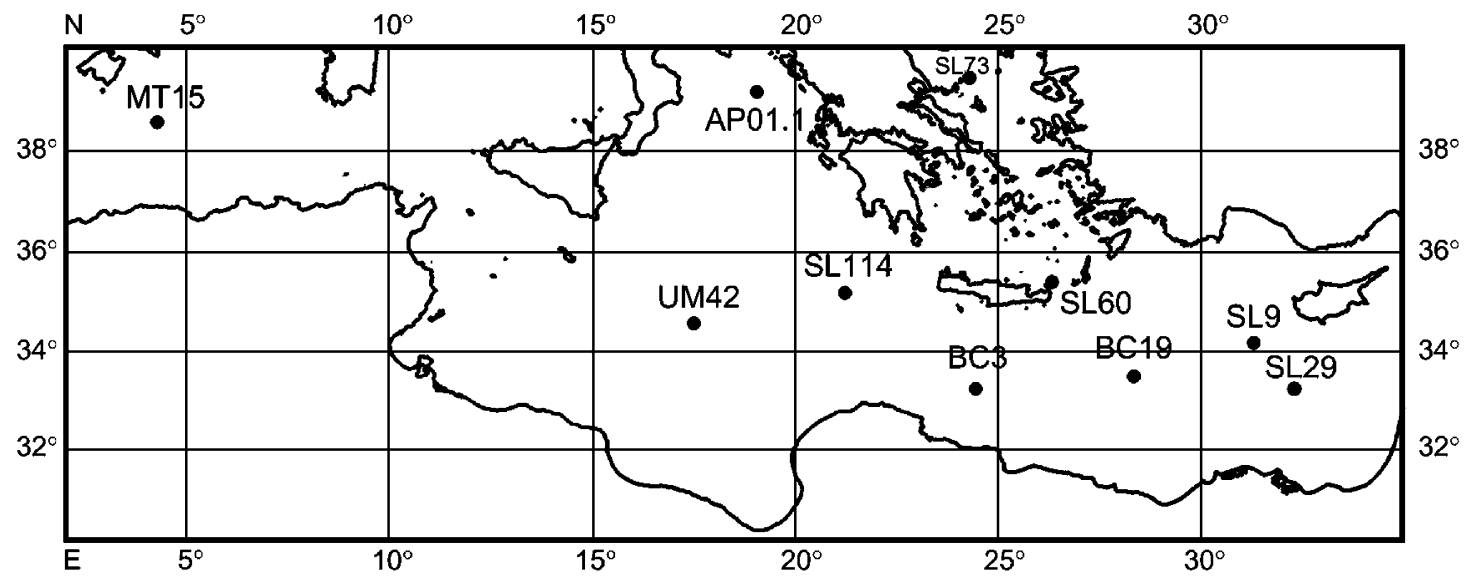

Fig. 1. Map of the Mediterranean Sea showing the locations of the analysed cores. 
ranean without visual evidence of the sapropel was analysed.

The depth of the unoxidised and oxidised portion of the sapropel (van Santvoort et al., 1996) recorded in cores from the eastern basin was traced on the basis of geochemical analyses (Thomson et al., 2004; de Lange et al., in prep.; de Lange, pers.com.). Details of tephra layers in the selected cores can be found in Principato (2003) and Crudeli et al. (2004).

Changes in zooplankton and phytoplankton assemblages across sapropels have been extensively studied (e.g., Castradori, 1993; Negri et al., 1999; Corselli et al., 2002; Principato, 2003; Principato et al., 2003; Giunta et al., 2003; Meier et al., 2004; Thomson et al., 2004). An ecostratigraphic scheme based on calcareous nannofossil has been proposed for the Holocene-late Pleistocene of the South Adriatic Sea (Giunta et al., 2003) and has been shown to be of regional value (Principato et al., 2003). Sapropel S1 was deposited during Interval 3 (Giunta et al., 2003) (ecozone C3, Principato et al., 2003) dated at $8.6 \mathrm{kyr}$ BP to 6.5 kyr BP (Giunta et al., 2003). Interval 3 is characterised by a consistent presence of $S$. pulchra and a peak in abundance of $H$. carteri. Similar patterns are documented in this work (S. pulchra HET and H. carteri HET in this work) and further support the nannofossil ecostratigraphy.

In the Western Mediterranean core MT15, the sedimentary interval co-eval with the deposition of the eastern Mediterranean sapropel is traced on the base of correlation between the foraminiferal assemblage (Principato, unpublished) and foraminifera biozones (Sbaffi et al., 2001).

Sapropel S1 is the only sapropel within the range of ${ }^{14} \mathrm{C}$ dating. In detail, for cores UM42, BC19, SL60 and SL9, a set of radiocarbon analyses from planktonic foraminifera were obtained in and around the S1 sapropel and used to convert depth in core to radiocarbon time (Mercone et al., 2000) (Thomson et al., 2004; Principato et al., in press, respectively) which are not reported in this work due to lack of similar chronology in the remaining cores.

\subsection{Light microscopy}

A total of 522 smear slides prepared according to standard techniques (e.g., Castradori, 1993) were analysed with a Wild Leitz light microscope (LM) at a magnification of $1250 \times$. The relative abundances of holococcoliths and relevant heterococcoliths were calculated by counting 100 to 150 calcareous nannofossils with the exclusion of Noelaerhabdaceae (Emiliania and
Gephyrocapsa) and lower photic-zone taxa (Florisphaera, Gladiolithus and Algirosphaera).

\subsection{Scanning electron microscopy}

Selected samples from cores BC3 and UM42 were qualitatively analysed by scanning electron microscopy (Philips XL30, Field Emission SEM) at the Natural History Museum, London and ca. 60 holococcoliths were imaged from these samples (Crudeli and Young, 2003). Images displayed in Fig. 6 were chosen to illustrate the state of preservation of holococcoliths.

\section{Light microscopy identification of holococcoliths and taxonomic remarks}

Light microscopic identification of holococcoliths reported in this work is based on Crudeli and Young (2003). The LM behavior of forms is summarized below. In this work, for S. pulchra and H. carteri and associated holococcolith phases, we adopt the revised taxonomy of Young et al. (2003), based on Cros et al. (2000), Geisen et al. (2002), Saugestad and Heimdal (2002) and Sáez et al. (2003). In particular we use the species names $S$. pulchra and $H$. carteri for both the holococcolith and heterococcolith phases of these species and add the informal terms HOL and HET to indicate the coccolith type observed. Cros et al. (2000) proposed use of the terms $\mathrm{HO}$ and $\mathrm{HE}$, to distinguish holococcolith and heterococcolith phases of a single species, Young et al. (2003) modified these to HOL and HET to make them clearer and easier to use in speech.

Two main types of holococcoliths are preserved in the sediments, calyptroliths and syracoliths. Calyptroliths are dome-shape holococcoliths (e.g., Kleijne, 1991; Young et al., 1997), and are characterised in the LM, by a narrow birefringent rim showing a radial extinction cross in plan view. We distinguished three types: S. pulchra HOL oblonga type (formerly known as Calyptrosphaera oblonga), which has a dark inner area; $S$. pulchra HOL pirus type (formerly known as Daktylethra pirus), which has a second birefringent ring within the inner area, and unclassified calyptroliths, which show variable characteristics. In the first cores studied, UM42, BC19 and BC3 only S. pulchra HOL oblonga type was separately distinguished whilst $S$. pulchra HOL pirus type was included in the unclassified calyptroliths.

Syracoliths are relatively solid holoccoliths formed of numerous superposed layers of crystallites (Kleijne, 1991). In LM the bulk of the coccolith appears to be formed of one or few relatively large pseudo-crystals or 
Table 2

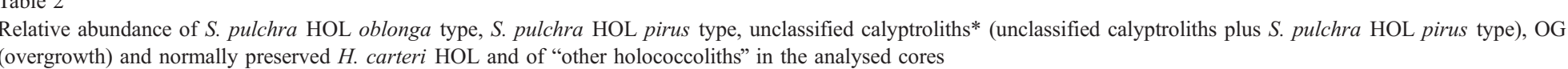
and normally preserved $H$. carteri $\mathrm{HOL}$ and of "other holococcoliths" in the analysed cores

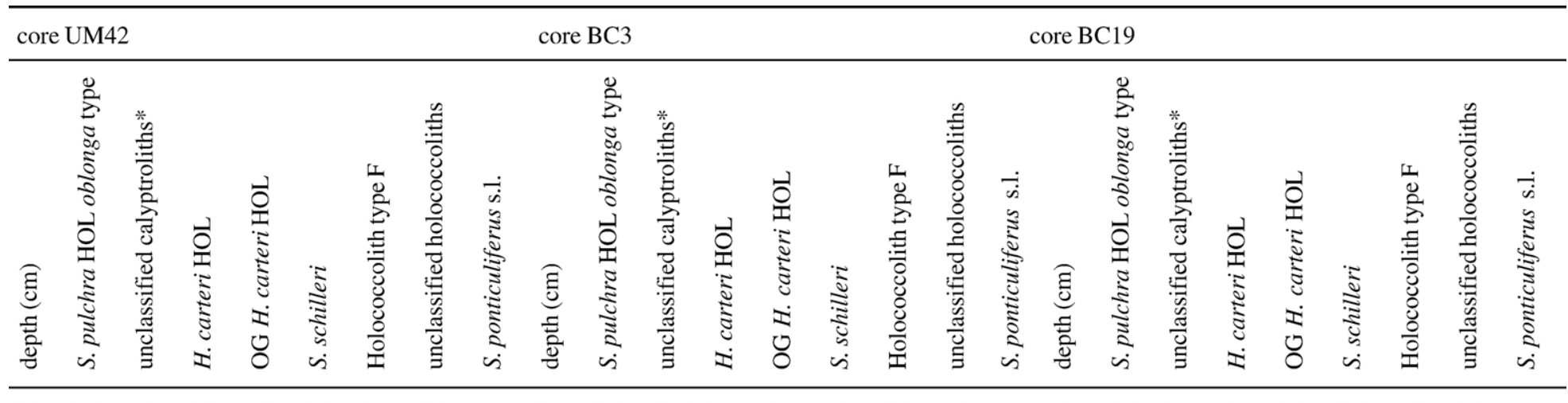

$\begin{array}{llllllll}0.7 & 7.4 & 8.3 & 1.9 & 1.9 & 0.0 & 0.9 & 1.9\end{array}$ $\begin{array}{lllllllll}2.2 & 8.5 & 5.7 & 0.0 & 0.0 & 0.0 & 0.0 & 0.0\end{array}$ $\begin{array}{lllllllll}3.2 & 9.2 & 10.0 & 2.3 & 1.5 & 0.0 & 0.0 & 1.5\end{array}$ $\begin{array}{lllllllll}4.2 & 6.3 & 11.0 & 1.6 & 1.6 & 0.8 & 0.8 & 2.4\end{array}$ $\begin{array}{llllllll}5.2 & 9.6 & 8.8 & 0.7 & 2.2 & 0.0 & 0.0 & 1.5\end{array}$ $\begin{array}{llllllll}6.2 & 7.3 & 12.9 & 2.4 & 0.8 & 0.0 & 1.6 & 1.6\end{array}$ $\begin{array}{llllllll}6.9 & 9.1 & 5.6 & 2.8 & 2.1 & 0.0 & 0.0 & 1.4\end{array}$ $\begin{array}{llllllll}7.7 & 10.6 & 16.3 & 0.0 & 3.8 & 0.0 & 0.0 & 0.0\end{array}$ $\begin{array}{llllllll}8.7 & 12.1 & 9.3 & 1.9 & 1.9 & 0.9 & 0.0 & 0.0\end{array}$ $\begin{array}{lllllllll}9.7 & 9.8 & 9.8 & 1.0 & 2.0 & 0.0 & 0.0 & 0.0\end{array}$ $\begin{array}{llllllllll}9.7 & 9.8 & 9.8 & 1.0 & 2.0 & 0.0 & 0.0 & 0.0 \\ 10.7 & 10.4 & 12.3 & 0.9 & 1.9 & 1.9 & 0.9 & 0.0 \\ 11.7 & 7.7 & 14.6 & 0.0 & 2.3 & 0.8 & 0.0 & 0.0\end{array}$ $\begin{array}{lllllllll}10.7 & 10.4 & 12.3 & 0.9 & 1.9 & 1.9 & 0.9 & 0.0 \\ 11.7 & 7.7 & 14.6 & 0.0 & 2.3 & 0.8 & 0.0 & 0.0 \\ 12.7 & 8.7 & 12.7 & 2.4 & 0.8 & 0.0 & 0.0 & 1.6\end{array}$ $\begin{array}{lllllllll}12.7 & 8.7 & 12.7 & 2.4 & 0.8 & 0.0 & 0.0 & 1.6\end{array}$ $\begin{array}{lllllllll}13.7 & 8.6 & 13.8 & 1.7 & 0.9 & 0.9 & 0.0 & 0.9\end{array}$ $\begin{array}{lllllllll}14.7 & 7.9 & 14.0 & 1.8 & 1.8 & 0.0 & 0.9 & 0.9\end{array}$ $\begin{array}{lllllllll}15.7 & 4.9 & 6.8 & 0.0 & 3.9 & 0.0 & 0.0 & 1.0\end{array}$ $\begin{array}{llllllllll}16.7 & 6.9 & 8.8 & 1.0 & 1.0 & 0.0 & 0.0 & 2.0\end{array}$ $\begin{array}{llllllllllllllll}8.3 & 12.5 & 2.4 & 0.0 & 0.0 & 0.0 & 4.0 & \mathrm{p} & 0.5 & 213 & 10.0 & 3.8 & 5.0 & 2.5 & 1.3 & 1.3\end{array}$ $\begin{array}{lllllllll}15.2 & 9.0 & 13.5 & 3.6 & 0.0 & 0.0 & 0.0 & 1.8\end{array}$ \begin{tabular}{llllllll}
16.4 & 5.8 & 7.2 & 3.5 & 0.0 & 0.0 & 0.0 & 0.9 \\
\hline 17.6 & 2.5 & 7.0 & 2.9 & 0.0 & 0.0 & 0.7 & 1.5
\end{tabular} $\begin{array}{llllllll}17.6 & 2.5 & 7.0 & 2.9 & 0.0 & 0.0 & 0.7 & 1.5 \\ 18.8 & 3.7 & 0.9 & 3.7 & 0.9 & 1.9 & 0.9 & 3.7\end{array}$ $\begin{array}{llllllll}20.6 & 2.9 & 1.9 & 2.9 & 0.0 & 1.9 & 1.0 & 1.9\end{array}$ \begin{tabular}{llllllll}
21.8 & 5.0 & 2.0 & 3.0 & 1.0 & 0.0 & 1.0 & 4.0 \\
\hline 23 & 13.7 & 11.8 & 2.9 & 1.0 & 1.0 & 1.0 & 2.0
\end{tabular} $\begin{array}{lllllllll}23 & 13.7 & 11.8 & 2.9 & 1.0 & 1.0 & 1.0 & 2.0\end{array}$ $\begin{array}{llllllllllll}24.2 & 11.2 & 13.1 & 1.5 & 2.9 & 0.7 & 1.5 & 2.2\end{array}$ $\begin{array}{llllllllll}25.4 & 11.1 & 10.2 & 2.8 & 1.9 & 0.9 & 0.0 & 0.9\end{array}$ $\begin{array}{llllllllll}26.6 & 8.9 & 11.6 & 0.0 & 0.0 & 0.0 & 0.9 & 0.0\end{array}$ $\begin{array}{lllllllll}27.8 & 20.8 & 18.8 & 0.0 & 2.0 & 0.0 & 0.0 & 0.0 \\ 29 & 12.3 & 29.2 & 0.0 & 1.9 & 0.0 & 0.0 & 0.9\end{array}$ $\begin{array}{llllllll}30.2 & 16.7 & 15.7 & 2.9 & 1.0 & 0.0 & 0.0 & 1.0\end{array}$ $\begin{array}{lllllllll}31.4 & 11.5 & 14.2 & 0.0 & 0.0 & 0.7 & 0.7 & 0.0\end{array}$ $\begin{array}{lllllllll}32.6 & 15.6 & 10.1 & 1.8 & 1.8 & 1.8 & 0.0 & 0.9\end{array}$ $\begin{array}{lllllllll}33.8 & 16.9 & 3.1 & 1.5 & 3.8 & 0.0 & 0.0 & 1.5\end{array}$ $\begin{array}{llllllllll}35 & 18.8 & 8.9 & 0.0 & 4.0 & 1.0 & 1.0 & 1.0\end{array}$ $\begin{array}{lllllllllll}36.2 & 18.7 & 1.9 & 0.0 & 2.6 & 0.6 & 0.0 & 1.3\end{array}$ $\begin{array}{lllllllll}37.4 & 19.3 & 6.7 & 0.8 & 5.9 & 0.8 & 0.0 & 0.8 \\ 38.6 & 33.1 & 5.0 & 0.6 & 2.5 & 0.0 & 0.6 & 1.9\end{array}$ $\begin{array}{lllllllll}38.6 & 33.1 & 5.0 & 0.6 & 2.5 & 0.0 & 0.6 & 1.9 \\ 40.4 & 10.9 & 5.9 & 1.0 & 2.0 & 0.0 & 1.0 & 2.0\end{array}$ $\begin{array}{llllllll}40.4 & 10.9 & 5.9 & 1.0 & 2.0 & 0.0 & 1.0 & 2.0 \\ 41.6 & 7.8 & 2.0 & 1.0 & 4.9 & 0.0 & 0.0 & 1.0\end{array}$ $\begin{array}{llllllllll}42.8 & 13.7 & 4.9 & 0.0 & 2.0 & 0.0 & 0.0 & 1.0\end{array}$ $\begin{array}{lllllllll}43.4 & 5.5 & 1.8 & 0.0 & 4.5 & 0.9 & 0.0 & 0.0\end{array}$ $\begin{array}{lllllllll}44.6 & 10.9 & 5.9 & 0.0 & 2.0 & 1.0 & 1.0 & 1.0\end{array}$ $\begin{array}{lllllllll}45.8 & 5.9 & 2.0 & 1.0 & 2.0 & 0.0 & 0.0 & 1.0\end{array}$ $\begin{array}{llllllllll}45.8 & 5.9 & 2.0 & 1.0 & 2.0 & 0.0 & 0.0 & 1.0 \\ 47 & 10.9 & 2.0 & 0.0 & 2.0 & 1.0 & 0.0 & 1.0\end{array}$ $\begin{array}{llllllllll}47 & 10.9 & 2.0 & 0.0 & 2.0 & 1.0 & 0.0 & 1.0 \\ 48.2 & 13.4 & 0.9 & 0.9 & 0.0 & 0.0 & 0.0 & 0.9\end{array}$ $\begin{array}{rrrrrrrrr}48.2 & 13.4 & 0.9 & 0.9 & 0.0 & 0.0 & 0.0 & 0.9 \\ 49.4 & 11.9 & 9.9 & 0.0 & 3.0 & 0.0 & 0.0 & 0.0\end{array}$ $\begin{array}{lllllllll}49.4 & 11.9 & 9.9 & 0.0 & 3.0 & 0.0 & 0.0 & 0.0 \\ 50.6 & 5.7 & 3.8 & 0.0 & 2.8 & 0.0 & 0.0 & 0.0\end{array}$ $\begin{array}{llllllll}50.6 & 5.7 & 3.8 & 0.0 & 2.8 & 0.0 & 0.0 & 0.0 \\ 51.8 & 10.9 & 6.9 & 0.0 & 0.0 & 0.0 & 0.0 & 0.0\end{array}$ $\begin{array}{lllllllll}51.8 & 10.9 & 6.9 & 0.0 & 0.0 & 0.0 & 0.0 & 0.0\end{array}$ $\begin{array}{lllllllll}53 & 10.6 & 1.6 & 0.0 & 1.6 & 0.0 & 0.0 & 0.0\end{array}$ $\begin{array}{lllllllll}54.2 & 2.9 & 3.9 & 0.0 & 3.9 & 0.0 & 1.0 & 0.0\end{array}$ $\begin{array}{lllllllll}55.4 & 9.8 & 2.0 & 0.0 & 0.0 & 0.0 & 0.0 & 0.0\end{array}$ $\begin{array}{lllllllll}56.6 & 9.8 & 2.9 & 0.0 & 1.0 & 0.0 & 0.0 & 0.0\end{array}$ $\begin{array}{lllllllll}57.8 & 6.2 & 2.7 & 0.0 & 0.9 & 0.9 & 0.0 & 0.9\end{array}$ $\begin{array}{llllllllll}59 & 12.8 & 3.7 & 0.9 & 1.8 & 0.9 & 0.0 & 0.0 & \mathrm{p}\end{array}$ $\begin{array}{lllllllll}60.2 & 3.7 & 3.0 & 0.0 & 0.7 & 0.7 & 0.0 & 1.5\end{array}$ $\begin{array}{lllllllll}61.4 & 7.3 & 1.8 & 0.9 & 1.8 & 0.9 & 0.0 & 0.0\end{array}$ $\begin{array}{lllllllll}62.6 & 5.5 & 0.0 & 0.0 & 2.7 & 0.0 & 0.0 & 0.0\end{array}$ $\begin{array}{llllllllll}63.8 & 1.9 & 10.6 & 0.0 & 4.8 & 1.0 & 0.0 & 0.0\end{array}$ $\begin{array}{llllllllll}65 & 5.9 & 1.9 & 106 & 0.0 & 4.8 & 1.0 & 0.0 & 0.0\end{array}$ $\begin{array}{llllllllll}66.2 & 10.2 & 1.9 & 0.0 & 1.9 & 0.9 & 0.0 & 0.0 & \mathrm{p}\end{array}$ $\begin{array}{lllllllllll}66.2 & 10.2 & 1.9 & 0.0 & 1.9 & 0.9 & 0.0 & 0.0\end{array}$ $\begin{array}{lllllllllllll}67.4 & 14.2 & 0.0 & 0.0 & 0.0 & 0.0 & 0.0 & 2.7\end{array}$ $\begin{array}{lllllllll}68.6 & 9.6 & 5.6 & 0.0 & 1.6 & 0.8 & 0.0 & 0.0\end{array}$ $\begin{array}{lllllllll}69.8 & 1.9 & 4.3 & 0.6 & 0.6 & 0.6 & 1.9 & 0.6\end{array}$ $\begin{array}{lllllllll}71 & 5.9 & 5.0 & 2.0 & 3.0 & 0.0 & 0.0 & 0.0\end{array}$ $\begin{array}{lllllllll}72.8 & 6.1 & 2.3 & 2.3 & 0.0 & 0.0 & 0.8 & 1.5\end{array}$ $\begin{array}{lllllllll}74 & 5.9 & 2.9 & 2.0 & 3.9 & 0.0 & 0.0 & 0.0\end{array}$ $\begin{array}{lllllllll}75.2 & 7.2 & 3.6 & 0.6 & 2.4 & 0.6 & 0.0 & 0.0\end{array}$ $\begin{array}{llllllllll}76.4 & 10.3 & 7.5 & 0.9 & 4.7 & 0.0 & 0.0 & 0.9\end{array}$ $\begin{array}{llllllllllll}76.4 & 10.3 & 7.5 & 0.9 & 4.7 & 0.0 & 0.0 & 0.9\end{array}$ $\begin{array}{lllllllllll}77.6 & 7.9 & 2.6 & 1.3 & 0.4 & 0.0 & 0.4 & 0.9\end{array}$ $\begin{array}{llllllllll}79.4 & 6.5 & 2.8 & 3.7 & 4.6 & 0.0 & 0.0 & 1.9\end{array}$ $81.2 \begin{array}{lllllllll}83.0 & 0.0 & 2.6 & 0.0 & 0.0 & 0.0 & 0.0\end{array}$ $\begin{array}{lllllllll}82.4 & 3.0 & 4.0 & 1.0 & 5.0 & 0.0 & 0.0 & 1.0\end{array}$ $\begin{array}{lllllllll}83.6 & 3.9 & 0.0 & 2.9 & 1.0 & 0.0 & 0.0 & 1.0\end{array}$ $\begin{array}{lllllllll}84.8 & 4.5 & 2.7 & 0.0 & 3.6 & 0.0 & 0.9 & 0.0\end{array}$

$\begin{array}{llllllllll}86 & 2.9 & 20 & 0.0 & 20 & 0.0 & 0.0 & 0.0\end{array}$

$\begin{array}{lllllllll}1.5 & 20.0 & 17.6 & 3.5 & 2.4 & 1.2 & 1.2 & 1.2\end{array}$ $\begin{array}{lllllllll}2.5 & 20.7 & 17.1 & 0.0 & 1.2 & 0.0 & 0.0 & 1.2\end{array}$ $\begin{array}{llllllll}3.5 & 25.0 & 21.1 & 0.0 & 1.3 & 1.3 & 1.3 & 0.0\end{array}$ $\begin{array}{llllllllll}4.5 & 26.5 & 24.1 & 1.2 & 1.2 & 0.0 & 1.2 & 0.0\end{array}$ $\begin{array}{lllllllll}5.5 & 27.7 & 21.7 & 0.0 & 1.2 & 1.2 & 1.2 & 0.0\end{array}$ $\begin{array}{llllllllll}6.5 & 25.7 & 24.3 & 0.0 & 2.7 & 1.4 & 1.4 & 0.0\end{array}$ $\begin{array}{lllllllll}7.75 & 23.7 & 27.6 & 0.0 & 2.6 & 0.0 & 0.0 & 0.0\end{array}$ $\begin{array}{lllllllll}9 & 10.3 & 20.6 & 1.0 & 2.1 & 0.0 & 0.0 & 1.0\end{array}$ $\begin{array}{llllllllll}9.75 & 14.3 & 14.3 & 3.1 & 3.1 & 0.0 & 0.0 & 0.0\end{array}$ $\begin{array}{lllllllll}10.5 & 28.6 & 22.9 & 0.0 & 1.4 & 0.0 & 0.0 & 0.0\end{array}$ $\begin{array}{llllllllll}11.5 & 28.4 & 20.3 & 0.0 & 2.7 & 0.0 & 1.4 & 1.4\end{array}$ $\begin{array}{llllllll}12.5 & 9.4 & 8.3 & 1.0 & 1.0 & 0.0 & 2.1 & 1.0\end{array}$ $\begin{array}{lllllllll}13.5 & 20.0 & 13.3 & 1.3 & 1.3 & 0.0 & 0.0 & 2.7\end{array}$ $\begin{array}{lllllllll}14.5 & 12.8 & 15.1 & 2.3 & 1.2 & 1.2 & 2.3 & 1.2\end{array}$

\begin{tabular}{llllllll}
15.5 & 7.0 & 9.0 & 1.0 & 0.0 & 0.0 & 0.0 & 2.0 \\
\hline 16.5 & 6.0 & 9.0 & 0.0 & 0.0 & 0.0 & 0.0 & 3.0
\end{tabular} $\begin{array}{lllllllllll}17.5 & 10 & 70 & 20 & 0.0 & 0.0 & 0.0 & 3.0\end{array}$

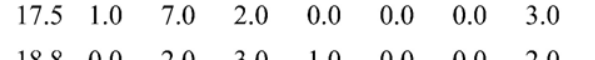
$\begin{array}{llllllllllll}18.5 & 1.0 & 7.0 & 2.0 & 0.0 & 0.0 & 0.0 & 3.0\end{array}$ $\begin{array}{lllllllllll}20 & 0.0 & 0.0 & 4.0 & 1.0 & 0.0 & 0.0 & 3.0\end{array}$ $\begin{array}{lllllllll}21 & 0.0 & 0.0 & 2.0 & 0.0 & 0.0 & 0.0 & 3.0\end{array}$ \begin{tabular}{llllllll}
22 & 0.0 & 0.0 & 2.0 & 0.0 & 0.0 & 0.0 & 2.0 \\
\hline 23 & 0.0 & 0.0 & 2.0 & 0.0 & 0.0 & 0.0 & 2.0
\end{tabular} $\begin{array}{llllllllll}24 & 0.0 & 0.0 & 1.0 & 0.0 & 0.0 & 0.0 & 1.0\end{array}$ $\begin{array}{lllllllll}25 & 0.0 & 0.0 & 1.0 & 0.0 & 0.0 & 0.0 & 2.0\end{array}$ $\begin{array}{lllllllll}26 & 0.0 & 0.0 & 0.0 & 0.0 & 0.0 & 0.0 & 0.0\end{array}$ $\begin{array}{lllllllllll}26 & 0.0 & 0.0 & 0.0 & 0.0 & 0.0 & 0.0 & 0.0\end{array}$ \begin{tabular}{llllllll}
28 & 5.0 & 12.0 & 7.0 & 0.0 & 0.0 & 1.0 & 0.0 \\
\hline 29 & 8.0 & 8.0 & 5.0 & 0.0 & 0.0 & 1.0 & 2.0
\end{tabular} $\begin{array}{lllllllll}30 & 13.1 & 26.3 & 2.0 & 0.0 & 0.0 & 0.0 & 3.0 \\ 31 & 14.3 & 11.2 & 5.1 & 0.0 & 1.0 & 0.0 & 2.0\end{array}$ $\begin{array}{llllllllll}31 & 14.3 & 11.2 & 5.1 & 0.0 & 1.0 & 0.0 & 2.0\end{array}$ $\begin{array}{llllllll}32 & 7.0 & 8.0 & 2.0 & 2.0 & 2.0 & 1.0 & 3.0\end{array}$ $\begin{array}{lllllllll}33.4 & 6.0 & 9.0 & 2.0 & 2.0 & 1.0 & 1.0 & 2.0\end{array}$ $\begin{array}{lllllllllllll}34.7 & 138 & 128 & 11 & 2.1 & 0.0 & 0.0 & 2.0\end{array}$ 


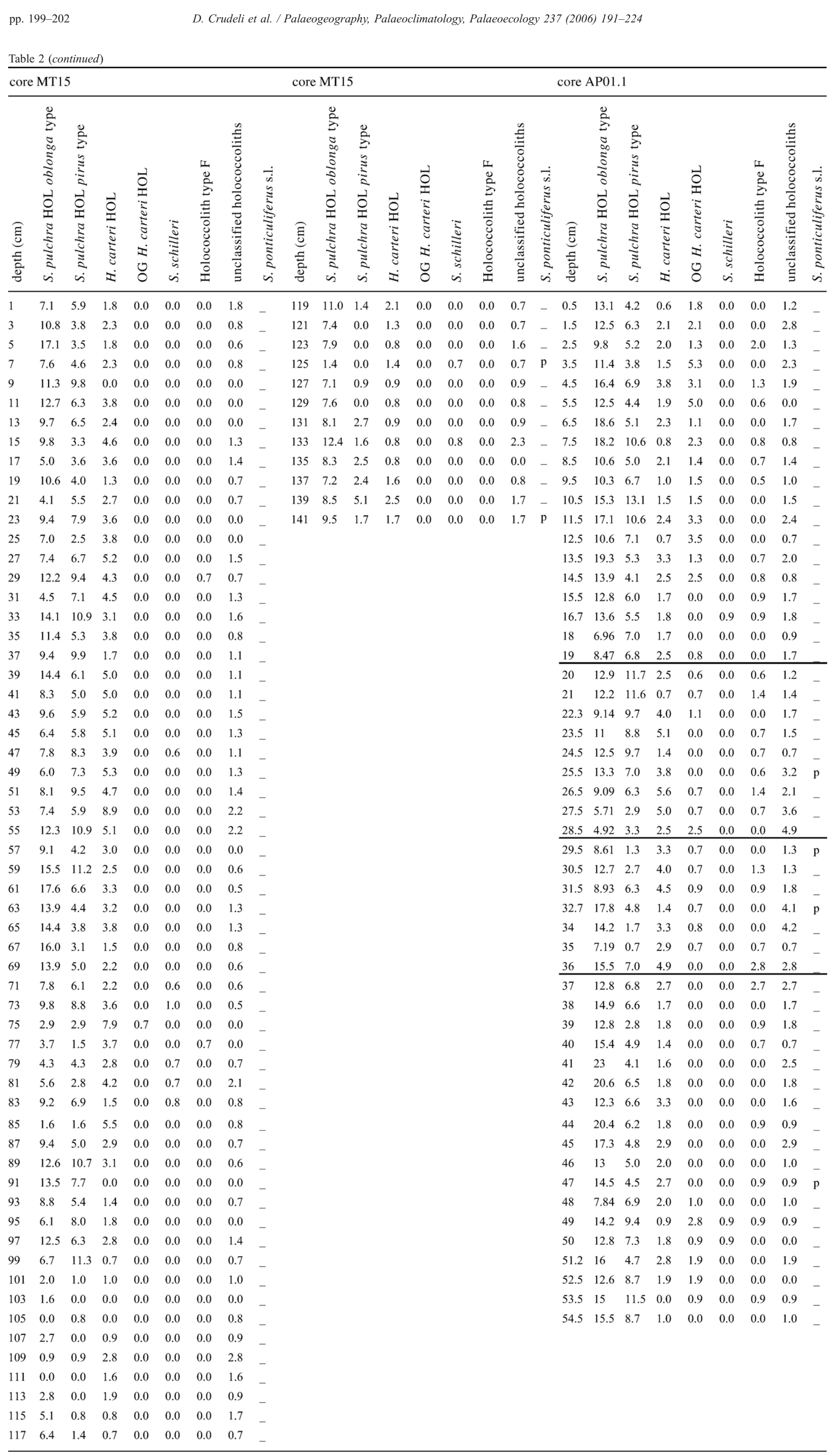




\begin{tabular}{|c|c|c|c|c|c|c|c|c|c|c|c|c|c|c|c|c|c|c|c|c|c|c|c|c|c|c|}
\hline \multicolumn{8}{|c|}{ core SL114 } & \multicolumn{8}{|c|}{ core SL73 } & \multicolumn{11}{|c|}{ core SL60 } \\
\hline 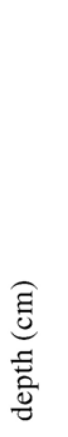 & 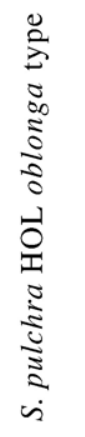 & 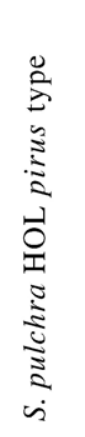 & 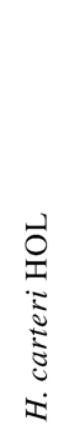 & 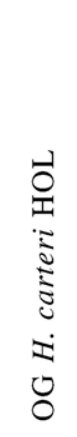 & 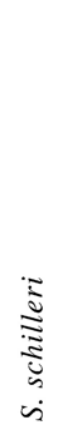 & 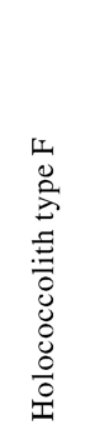 & 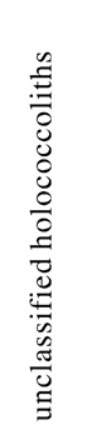 & 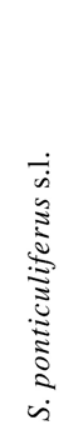 & 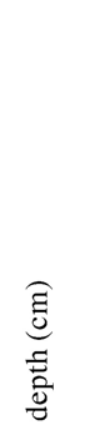 & 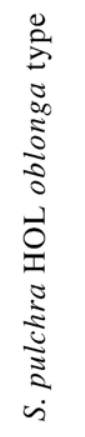 & 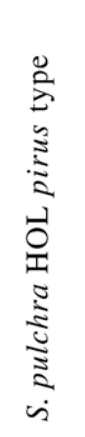 & 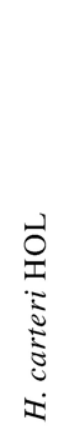 & 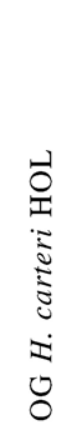 & 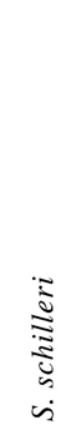 & 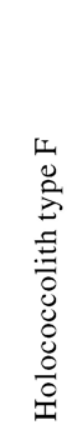 & 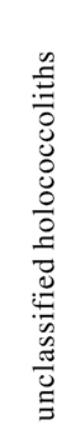 & 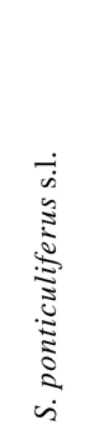 & $\begin{array}{l}\text { 言 } \\
\text { 䇏 } \\
\text { 喜 }\end{array}$ & 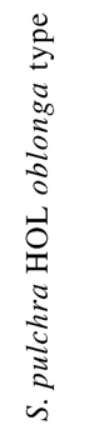 & 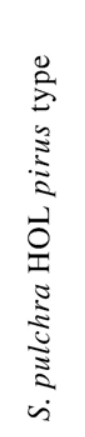 & 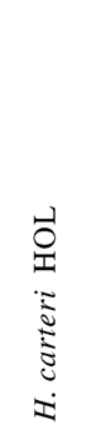 & 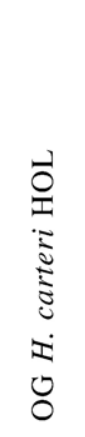 & 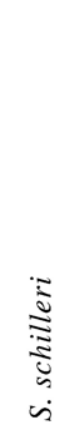 & 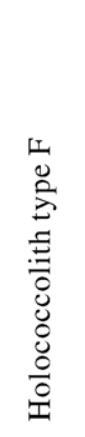 & 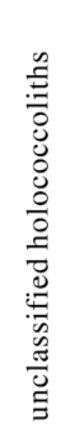 & 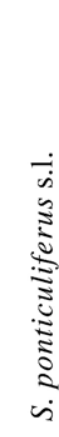 \\
\hline 0.5 & 16.5 & 3.7 & 2.2 & 0.4 & 0.4 & 0.0 & 0.7 & - & 0.9 & 15.9 & 8.2 & 1.2 & 0.6 & 0.0 & 0.0 & 1.2 & _ & 1.5 & 16.2 & 6.0 & 1.8 & 1.2 & 0.0 & 0.0 & 0.0 & _- \\
\hline 1.5 & 11.5 & 4.5 & 1.9 & 0.6 & 0.6 & 0.6 & 1.9 & _ & 1.9 & 15.0 & 7.8 & 1.2 & 0.0 & 0.0 & 0.0 & 2.4 & _ & 4 & 12.3 & 7.7 & 2.3 & 1.5 & 0.0 & 0.0 & 0.0 & \\
\hline 2.5 & 9.0 & 6.0 & 0.5 & 0.5 & 0.5 & 0.0 & 1.5 & - & 3.4 & 14.9 & 7.1 & 0.6 & 0.0 & 0.0 & 0.6 & 1.3 & - & 6 & 9.6 & 6.4 & 1.3 & 5.1 & 0.6 & 0.0 & 0.0 & - \\
\hline 3.5 & 14.9 & 9.3 & 0.6 & 0.0 & 0.0 & 0.0 & 1.9 & - & 4.4 & 20.1 & 6.3 & 1.3 & 0.0 & 0.0 & 0.0 & 1.3 & _ & 8 & 14.3 & 9.3 & 2.9 & 4.3 & 0.0 & 0.0 & 1.4 & $\mathrm{p}$ \\
\hline 4.5 & 15.0 & 13.3 & 1.1 & 0.6 & 0.0 & 0.0 & 0.6 & _- & 5.4 & 19.3 & 9.1 & 2.3 & 0.0 & 0.0 & 0.0 & 1.1 & _ & 10 & 14.8 & 6.8 & 2.5 & 0.0 & 0.0 & 0.6 & 0.6 & _- \\
\hline 5.5 & 15.5 & 10.7 & 0.4 & 1.7 & 0.9 & 0.4 & 1.7 & - & 6.4 & 24.4 & 9.6 & 1.5 & 0.0 & 0.5 & 0.0 & 0.5 & - & 12 & 12.6 & 9.9 & 1.3 & 0.0 & 0.0 & 0.0 & 0.0 & - \\
\hline 6.8 & 11.8 & 10.7 & 0.0 & 1.1 & 1.6 & 0.5 & 2.1 & - & 7.4 & 12.7 & 10.1 & 1.3 & 0.0 & 0.0 & 0.0 & 1.3 & _ & 14 & 12.8 & 7.4 & 1.3 & 5.4 & 0.0 & 0.7 & 0.7 & - \\
\hline 7.8 & 14.5 & 9.1 & 0.0 & 1.6 & 0.0 & 0.5 & 1.1 & - & 8.4 & 10.9 & 10.3 & 1.1 & 0.0 & 0.0 & 0.0 & 1.1 & _ & 16 & 12.8 & 11.5 & 2.0 & 4.1 & 0.7 & 0.0 & 0.7 & - \\
\hline 8.8 & 7.5 & 9.0 & 1.5 & 1.5 & 1.5 & 0.5 & 1.5 & - & 9.4 & 11.2 & 5.8 & 2.4 & 0.0 & 0.0 & 0.0 & 1.0 & _- & 18 & 13.6 & 5.9 & 0.6 & 0.0 & 0.6 & 0.0 & 0.6 & - \\
\hline 9.8 & 9.5 & 10.7 & 0.0 & 2.4 & 0.6 & 0.0 & 1.8 & _ & 10.4 & 6.9 & 5.8 & 1.7 & 0.0 & 0.0 & 0.0 & 1.7 & _ & 20 & 12.6 & 7.3 & 2.6 & 1.0 & 0.0 & 0.0 & 0.5 & _- \\
\hline 10.8 & 12.7 & 7.6 & 1.3 & 0.6 & 0.0 & 0.0 & 1.3 & _- & 11.9 & 12.9 & 6.7 & 0.6 & 0.0 & 0.0 & 0.0 & 0.0 & _ & 22 & 10.1 & 4.7 & 1.6 & 1.6 & 0.0 & 0.0 & 1.6 & - \\
\hline 11.8 & 13.6 & 8.5 & 0.6 & 2.3 & 0.0 & 2.3 & 0.6 & - & 12.9 & 9.8 & 4.9 & 0.0 & 0.0 & 0.0 & 0.0 & 0.5 & _ & 24 & 10.9 & 9.4 & 2.2 & 1.4 & 0.0 & 0.0 & 0.7 & - \\
\hline 12.8 & 14.5 & 13.5 & 1.8 & 0.7 & 0.4 & 0.4 & 0.4 & _ & 13.9 & 10.8 & 4.2 & 1.2 & 0.0 & 0.0 & 0.0 & 0.6 & _ & 26 & 13.0 & 6.5 & 1.8 & 1.2 & 0.0 & 0.0 & 0.0 & $\mathrm{p}$ \\
\hline 13.8 & 14.6 & 12.6 & 0.8 & 0.4 & 0.4 & 0.0 & 0.0 & _ & 14.9 & 8.6 & 6.4 & 1.1 & 0.5 & 0.5 & 0.0 & 0.5 & _ & 28 & 12.9 & 7.1 & 3.2 & 2.6 & 0.0 & 0.6 & 0.6 & $\mathrm{p}$ \\
\hline 14.8 & 11.2 & 12.1 & 0.9 & 0.3 & 0.3 & 0.3 & 0.3 & - & 15.9 & 14.5 & 6.6 & 0.6 & 0.0 & 0.6 & 0.6 & 0.6 & _ & 30 & 12.2 & 3.3 & 0.8 & 0.0 & .0 & 1.6 & .6 & - \\
\hline 15.8 & 13.7 & 13.0 & 1.1 & 1.5 & 0.4 & 0.4 & 0.0 & _ & 16.9 & 12.3 & 4.8 & 1.1 & 0.0 & 0.5 & 0.0 & 0.0 & _. & 32 & 12.4 & 7.0 & 1.6 & 0.0 & 0.0 & 0.0 & 1.6 & - \\
\hline 16.8 & 10.4 & 11.7 & 0.4 & 1.7 & 0.4 & 1.3 & 0.4 & - & 17.9 & 14.3 & 7.5 & 1.2 & 0.0 & 0.0 & 0.0 & 1.9 & $P$ & 34 & 12.8 & 7.8 & 0.7 & 0.0 & 0.7 & 0.0 & 0.7 & - \\
\hline 17.8 & 13.0 & 11.3 & 0.8 & 0.8 & 0.4 & 0.8 & 0.0 & _ & 18.9 & 7.4 & 3.7 & 1.1 & 0.0 & 0.0 & 0.0 & 1.1 & _ & 36 & 15.1 & 4.0 & 1.6 & 3.2 & 0.8 & 0.0 & .8 & - \\
\hline 18.8 & 6.1 & 12.3 & 1.6 & 0.8 & 0.4 & 0.4 & 0.0 & _ & 19.9 & 10.5 & 5.3 & 2.1 & 0.0 & 0.0 & 0.0 & 1.1 & 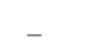 & 38 & 11.2 & 4.6 & 0.0 & 1.3 & 0.7 & 0.7 & 0.7 & - \\
\hline 19.8 & 3.0 & 4.9 & 3.0 & 0.0 & 1.1 & 0.8 & 0.8 & - & 20.9 & 8.3 & 5.4 & 2.4 & 0.0 & 0.6 & 0.0 & 0.6 & _ & 40 & 10.8 & 4.3 & 1.1 & 0.0 & 0.0 & 0.0 & 3.2 & - \\
\hline 20.8 & 2.0 & 4.5 & 4.9 & 0.0 & 0.0 & 0.0 & 1.6 & 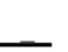 & 21.9 & 8.4 & 4.7 & 2.1 & 0.0 & 0.0 & 0.0 & 0.0 & 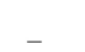 & 42 & 6.8 & 5.9 & 0.8 & 1.7 & 0.0 & 0.0 & .8 & 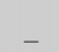 \\
\hline 21.8 & 1.4 & 3.6 & 2.3 & 0.0 & 0.0 & 0.9 & 1.8 & $\mathrm{p}$ & 22.9 & 9.5 & 4.1 & 2.4 & 0.6 & 0.6 & 0.0 & 0.6 & $P$ & 44 & 12.4 & 6.2 & 1.0 & 0.0 & 0.0 & 0.0 & .0 & - \\
\hline 22.8 & 0.5 & 0.9 & 4.1 & 0.5 & 0.0 & 0.0 & 0.9 & - & 23.9 & 11.2 & 5.9 & 2.7 & 0.0 & 0.0 & 0.0 & 0.5 & _ & 46 & 8.8 & 5.0 & 1.3 & 0.0 & 1.3 & 0.0 & 1.3 & - \\
\hline 23.8 & 0.0 & 0.0 & 2.8 & 0.0 & 0.0 & 0.5 & 0.9 & - & 24.9 & 11.7 & 3.7 & 1.9 & 0.0 & 0.6 & 0.0 & 1.2 & _ & 48 & 3.6 & 3.6 & 0.0 & 4.8 & 0.0 & 0.0 & .0 & _- \\
\hline 24.8 & 0.0 & 0.0 & 3.3 & 0.0 & 0.0 & 0.8 & 0.4 & - & 25.9 & 12.7 & 0.8 & 0.0 & 0.0 & 0.0 & 0.0 & 1.6 & - & 50 & 2.7 & 2.7 & 0.0 & 0.0 & 0.0 & 0.0 & .0 & - \\
\hline 25.8 & 0.0 & 0.0 & 2.1 & 0.0 & 0.0 & 0.0 & 0.8 & - & 27.5 & 12.0 & 3.0 & 0.0 & 0.0 & 0.0 & 0.0 & 0.0 & _ & 52 & 2.7 & 2.7 & 0.0 & 0.0 & 0.0 & 0.0 & 0.0 & - \\
\hline 27.3 & 0.0 & 0.0 & 1.6 & 0.0 & 0.4 & 0.8 & 0.8 & $\ldots$ & 28.5 & 6.8 & 2.3 & 1.5 & 0.0 & 0.0 & 0.0 & 1.5 & _ & 54 & 5.9 & 3.0 & 0.0 & 0.0 & 1.0 & 0.0 & 1.0 & - \\
\hline 28.3 & 0.0 & 0.0 & 3.5 & 0.0 & 0.0 & 0.0 & 0.9 & - & 29.5 & 11.0 & 2.4 & 0.8 & 0.0 & 2.4 & 0.0 & 0.8 & _ & 56.5 & 1.9 & 1.0 & 1.0 & 0.0 & 0.0 & 1.0 & .0 & \\
\hline 29.3 & 0.0 & 0.0 & 2.3 & 0.0 & 0.0 & 0.0 & 0.9 & _ & 30.5 & 9.9 & 1.4 & 1.4 & 0.0 & 0.0 & 0.0 & 1.4 & _ & 59 & 9.3 & 7.5 & 0.9 & 0.0 & 0.0 & 0.0 & .9 & - \\
\hline 30.3 & 0.0 & 0.8 & 3.1 & 0.0 & 0.0 & 0.0 & 0.8 & - & 31.5 & 6.0 & 0.9 & 2.6 & 0.0 & 0.0 & 0.0 & 1.7 & _ & 61 & 13.2 & 7.5 & 1.7 & 0.0 & 0.0 & 0.6 & 0.6 & - \\
\hline 31.3 & 0.9 & 0.5 & 2.3 & 0.0 & 0.0 & 0.0 & 0.9 & _ & 32.5 & 7.0 & 0.6 & 1.9 & 0.0 & 0.6 & 0.0 & 1.3 & _ & 63 & 18.5 & 6.8 & 2.1 & 0.0 & .7 & 17 & .4 & _- \\
\hline 32.3 & 2.6 & 2.1 & 3.6 & 0.0 & 0.0 & 0.0 & 1.0 & - & 33.5 & 3.7 & 0.7 & 2.2 & 0.0 & 0.0 & 0.0 & 1.5 & - & 65 & 13.3 & 8.9 & 1.3 & 1.3 & 1.3 & 0.0 & .3 & _- \\
\hline 33.8 & 3.0 & 1.8 & 4.2 & 0.0 & 0.0 & 0.6 & 1.2 & - & 34.5 & 6.7 & 2.2 & 3.0 & 0.0 & 0.7 & 0.0 & 2.2 & _ & 67 & 9.7 & 5.4 & 2.2 & 2.2 & 0.0 & 0.0 & .0 & - \\
\hline 34.8 & 4.5 & 2.9 & 2.5 & 0.4 & 0.4 & 0.0 & 2.1 & _ & 35.5 & 4.6 & 1.9 & 0.9 & 0.0 & 0.9 & 0.0 & 0.0 & 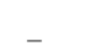 & 69 & 10.1 & 8.8 & 2.6 & 0.9 & 0.0 & 0.0 & .4 & _- \\
\hline 35.8 & 4.0 & 4.4 & 2.6 & 0.0 & 0.0 & 0.7 & 2.9 & & 36.5 & 0.0 & 8.3 & 1.3 & 0.0 & 0.0 & 0.6 & 1.9 & - & 71 & 5.7 & 7.5 & 2.8 & 0.0 & 0.0 & 0.0 & .9 & - \\
\hline 36.8 & 17.0 & 10.5 & 5.0 & 0.0 & 1.0 & 0.5 & 2.0 & $\mathrm{p}$ & 37.5 & 0.0 & 12.9 & 0.6 & 0.0 & 0.0 & 0.0 & 1.2 & - & 73 & 13.1 & 10.6 & 3.0 & 1.0 & 0.0 & 0.5 & 2.0 & - \\
\hline 37.8 & 12.0 & 6.0 & 2.3 & 0.3 & 0.3 & 0.3 & 1.3 & _ & 38.5 & 0.0 & 7.8 & 0.0 & 0.0 & 0.0 & 0.0 & 0.0 & 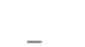 & 75 & 11.6 & 6.6 & 2.5 & 1.0 & 0.0 & 0.5 & 1.0 & \\
\hline 38.8 & 9.1 & 6.0 & 3.5 & 0.4 & 0.0 & 0.0 & 2.1 & - & & & & & & & & & & 77 & 10.9 & 7.5 & 4.0 & 1.1 & 0.0 & 0.0 & .1 & - \\
\hline 39.8 & 10.8 & 6.6 & 4.7 & 0.9 & 0.0 & 0.0 & 2.8 & _. & & & & & & & & & & 79 & 8.2 & 5.4 & 2.2 & 2.2 & .0 & 0.0 & .1 & - \\
\hline 40.8 & 9.1 & 5.7 & 4.8 & 1.0 & 0.5 & 0.5 & 1.4 & - & & & & & & & & & & 81 & 6.5 & 4.3 & 3.8 & 0.0 & 0.5 & 0.0 & 0.5 & $\mathrm{p}$ \\
\hline 41.8 & 11.7 & 4.9 & 4.9 & 0.5 & 0.5 & 0.0 & 2.9 & - & & & & & & & & & & 83 & 11.2 & 7.3 & 2.4 & 0.0 & .0 & 0.5 & 1.5 & $\mathrm{p}$ \\
\hline 42.8 & 18.2 & 7.3 & 3.6 & 0.9 & 0.0 & 0.0 & 1. & _ & & & & & & & & & & 85 & 10.7 & 7.3 & 1.3 & 0.0 & 0.4 & 0.9 & 0.0 & \\
\hline 43.8 & 18.1 & 8.0 & 2.5 & 0.5 & 1.5 & 1.0 & 0.5 & - & & & & & & & & & & 87 & 12.1 & 6.0 & 2.7 & 0.0 & 0.0 & 0.0 & .0 & - \\
\hline 44.8 & 13.5 & 8.1 & 0.4 & 0.0 & 0.4 & 0.9 & 0.9 & & & & & & & & & & & 89 & 8.8 & 7.7 & 4.1 & 0.0 & 0.0 & 0.0 & .5 & - \\
\hline 45.8 & 16.6 & 8.5 & 1.9 & 0.4 & 0.0 & 0.0 & 1.5 & & & & & & & & & & & 91 & 11.2 & 11.7 & 4.1 & 0.0 & 0.0 & 0.0 & 1.0 & \\
\hline 46.9 & 22.0 & 4.9 & 1.5 & 0.5 & 0.0 & 0.0 & 5 & - & & & & & & & & & & 93 & 8.7 & 3.7 & 4.3 & 0.0 & 0.6 & 0.6 & .2 & _- \\
\hline 47.9 & 19.9 & 3.0 & 1.2 & 1.2 & 0.6 & 0.6 & 1.2 & & & & & & & & & & & 95 & 11.1 & 5.3 & 2.9 & 1.2 & .6 & .0 & .8 & - \\
\hline \multirow{12}{*}{ 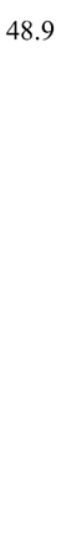 } & 21.8 & 2.9 & 1.1 & 2.3 & 0.6 & 0.6 & 1.1 & & & & & & & & & & & 97 & 13.5 & 5.7 & 4.7 & 0.0 & 0.5 & 0.0 & 1.6 & _- \\
\hline & & & & & & & & & & & & & & & & & & 99 & 11.9 & 6.2 & 5.7 & 0.0 & 0.0 & 0.0 & .1 & _ \\
\hline & & & & & & & & & & & & & & & & & & 102 & 7.2 & 6.6 & 4.8 & 0.0 & 0.0 & 0.0 & 1.2 & \\
\hline & & & & & & & & & & & & & & & & & & 104 & 15.4 & 14.5 & 2.3 & 0.0 & 0.5 & 0.5 & .4 & - \\
\hline & & & & & & & & & & & & & & & & & & 106 & 12.5 & 13.1 & 4.2 & 0.0 & 0.0 & 10 & .6 & - \\
\hline & & & & & & & & & & & & & & & & & & 108 & 18.4 & 13.5 & 1.8 & 1.2 & 0.6 & 0.0 & .6 & - \\
\hline & & & & & & & & & & & & & & & & & & 110 & 17.5 & 5.2 & 1.9 & 0.0 & 0.9 & 0.0 & .9 & - \\
\hline & & & & & & & & & & & & & & & & & & 11 & 13.2 & 5.9 & 2.5 & 0.0 & 1.0 & 0.0 & .5 & - \\
\hline & & & & & & & & & & & & & & & & & & 11 & 9. & 2.7 & 1.1 & 0 & 0.0 & 0.0 & 5 & - \\
\hline & & & & & & & & & & & & & & & & & & 116 & 15.1 & 3.1 & 1.9 & 0.0 & 0.6 & 0.0 & 9 & _ \\
\hline & & & & & & & & & & & & & & & & & & 11 & 15.6 & 11.3 & 1.1 & 1.1 & 0.0 & 0.0 & .5 & _ \\
\hline & & & & & & & & & & & & & & & & & & 120 & 13.3 & 8.3 & 2.2 & 1.1 & 0.6 & 0.6 & 0.0 & \\
\hline
\end{tabular}




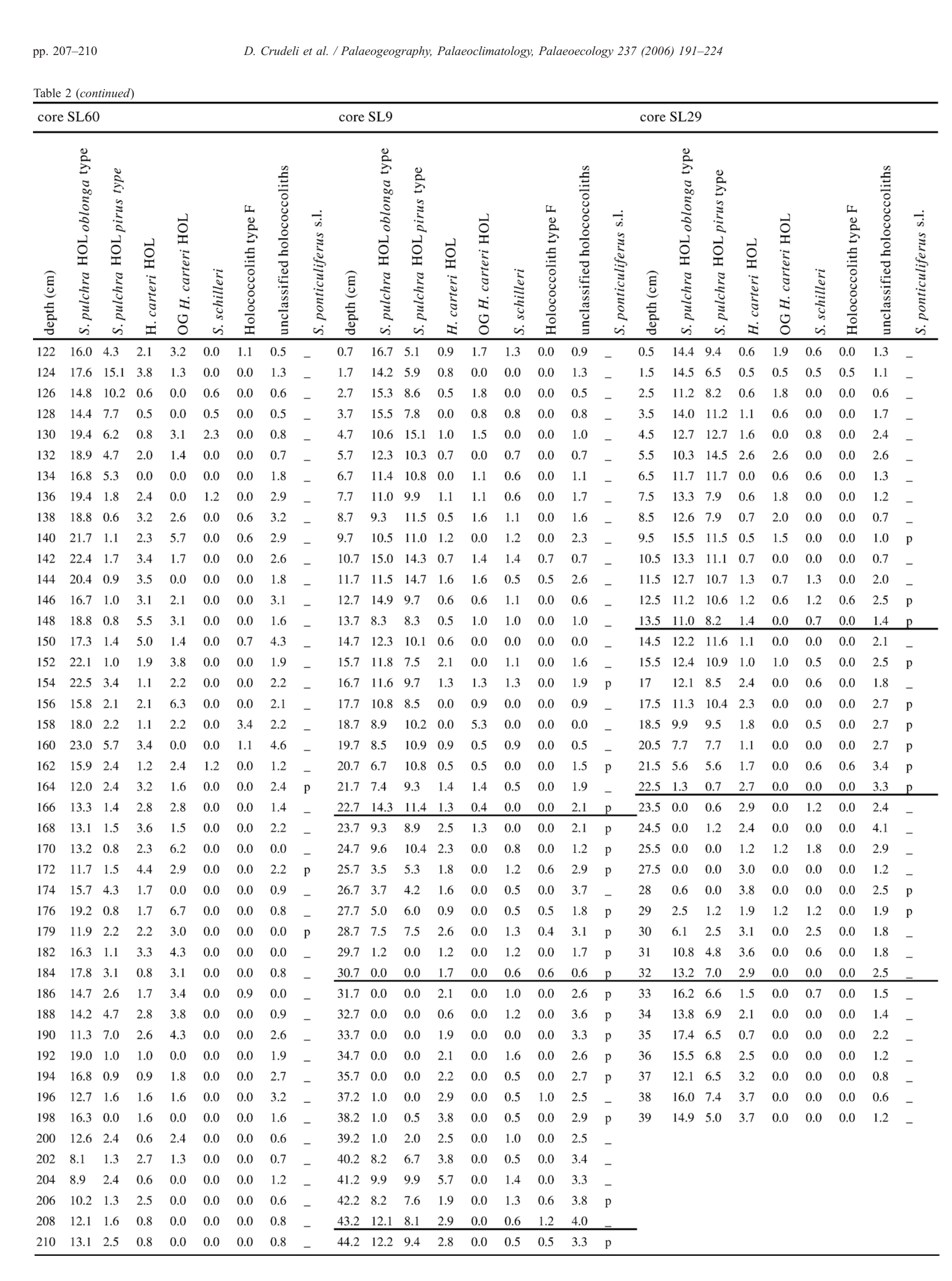


blocks (Crudeli and Young, 2003). The main syracoliths are $H$. carteri HOL solid (formerly known as Syracolithus catilliferus) and H. carteri HOL perforate (formerly known as Syracolithus confusus). These morphotypes represent a case of intraspecific variation in the degree of calcification of the holococcoliths (Cros et al., 2000; Geisen et al., 2002; Geisen et al., 2004). They were grouped together here and termed H. carteri HOL. We distinguished between overgrown and normally preserved H. carteri HOL (Crudeli and Young, 2003, Plate 2, Figs. 5-7). A similar distinction was too subjective for S. pulchra HOL and for unclassified calyptroliths because they showed complete variation in thickness-birefringence. H. carteri HOL is likely to include specimens of Syracolithus dalmaticus, which is possibly the holococcolith phase of Helicosphaera wallichii (Geisen et al., 2004).

Other holococcoliths observed in the sediments include Syracolithus schilleri, holococcolith type F (of Crudeli and Young, 2003) and Syracolithus ponticuliferus s.l. (S. ponticuliferus and/or Corisphaera/Zygosphaera) and other unidentified holococcoliths, mostly simple elliptical birefringent forms with variable numbers of perforations (Crudeli and Young, 2003).

\section{Results}

S. pulchra HOL oblonga type and S. pulchra HOL pirus type are combined here for comparison with the life-cycle stage $S$. pulchra HET and termed $S$. pulchra HOL (Young et al., 2003) (Table 2; Figs. 2, 3b, d, 4b, c and 5). In cores UM42, BC3, and $\mathrm{BC} 19$ the S. pulchra HOL oblonga type pattern is compared to $S$. pulchra HET since the pirus type was grouped with unclassified calyptroliths (Figs. 3a, c and 4a). H. carteri HET is compared with H. carteri HOL (Figs. 2-5). S. schilleri, holococcolith type F, $S$. ponticuliferus s.l. and unclassified holococcoliths are grouped and termed here "other holococcoliths" (Figs. 2-5). The relative abundance of these forms is summarised in Table 2.

\subsection{Relative abundance profiles}

\subsubsection{Core without visual evidence of sapropel S1 - Western Mediterranean}

In the Western Mediterranean core MT15, H. carteri HET shows higher abundance values than $S$. pulchra HET with a marked peak $(>35 \%$ of the coccolith assemblage excluding Noelaerhabdaceae and lower photic zone species) close to the base of core (Fig. 2) which correlate with. S. pulchra HOL have average abundances of about $15 \%$ above $100 \mathrm{~cm}$ depth whereas unclassified calyptroliths are absent. H. carteri HOL and "other holococcoliths" are less abundant and unclassified calyptroliths are absent. Cretaceous-Neogene reworked calcareous nannofossils are quite common in the assemblage close to the core bottom indicating reworking. The distribution pattern of Gephyrocapsa species and E. huxleyi from that core (Crudeli et al., 2004; Crudeli, unpublished) are roughly similar to that documented by Sbaffi et al. (2001) from other Western Mediterranean cores suggesting minor effect of reworking on assemblages.

\subsubsection{Cores with visual evidence of sapropel S1 - eastern Mediterranean}

4.1.2.1. S. pulchra HET-S. pulchra HOL. In sediments below sapropel S1 S. pulchra HET usually shows lower abundances than S. pulchra HOL which is very common (Figs. 3, 4 and 5a). Within S. pulchra HOL, the oblonga type normally dominates over the pirus type (Table 2). S. pulchra HET shows a slight increase in abundance within sapropel S1 (Figs. 3a, c-d and $4 \mathrm{~b}-\mathrm{c}$ ) decreasing upward. In the deeper cores, $S$. pulchra HOL decreases in the lower part of the sapropel, is absent in the central part of S1 and increases in abundance in the uppermost part. In contrast, in shallow cores AP01.1 and SL60 the group is present throughout the entire sapropel (Figs. 3d and 5a) whereas in Aegean Sea core SL73, it increases greatly in abundance upcore, from $<10 \%$ to $>20 \%$ (Fig. 5b). Above S1, both phases of $S$. pulchra have relative abundances similar to those in the pre-sapropel interval. Some overgrown Syracosphaera heterococcoliths are present outside the sapropel (Principato et al., in press). In cores UM42, BC3 and BC19 S. pulchra HOL oblonga type shows relative abundance values similar to those in most other cores (compare Figs. 3a, c and $4 \mathrm{a}$ and e.g., Fig. 3b). It is absent in a short interval within the sapropel of cores UM42 and BC19 and it has a relative abundance $<2.5 \%$ in $\mathrm{S} 1$ of core $\mathrm{BC} 3$.

4.1.2.2. H. carteri HET-H. carteri HOL. Below S1, $H$. carteri $\mathrm{HET}$ and $S$. pulchra $\mathrm{HET}$ have similar relative abundances whereas $H$. carteri HOL is consistently lower in abundance (average, $\sim 3 \%$ ) than S. pulchra HOL (Figs. 3-5). Within $\mathrm{S} 1$ in all cores, H. carteri HET shows two abundance peaks. Towards the core tops, it decreases again. In detail, in core SL73 H. carteri HET decreases in abundance from bottom to top being very rare close to the top. Within the sapropel $H$. carteri HOL is commonly $<5 \%$, occasionally $5 \%$ in abundance. Overgrown $H$. carteri $\mathrm{HOL}$ are variably present below 


\section{MT15 (2373 m)}

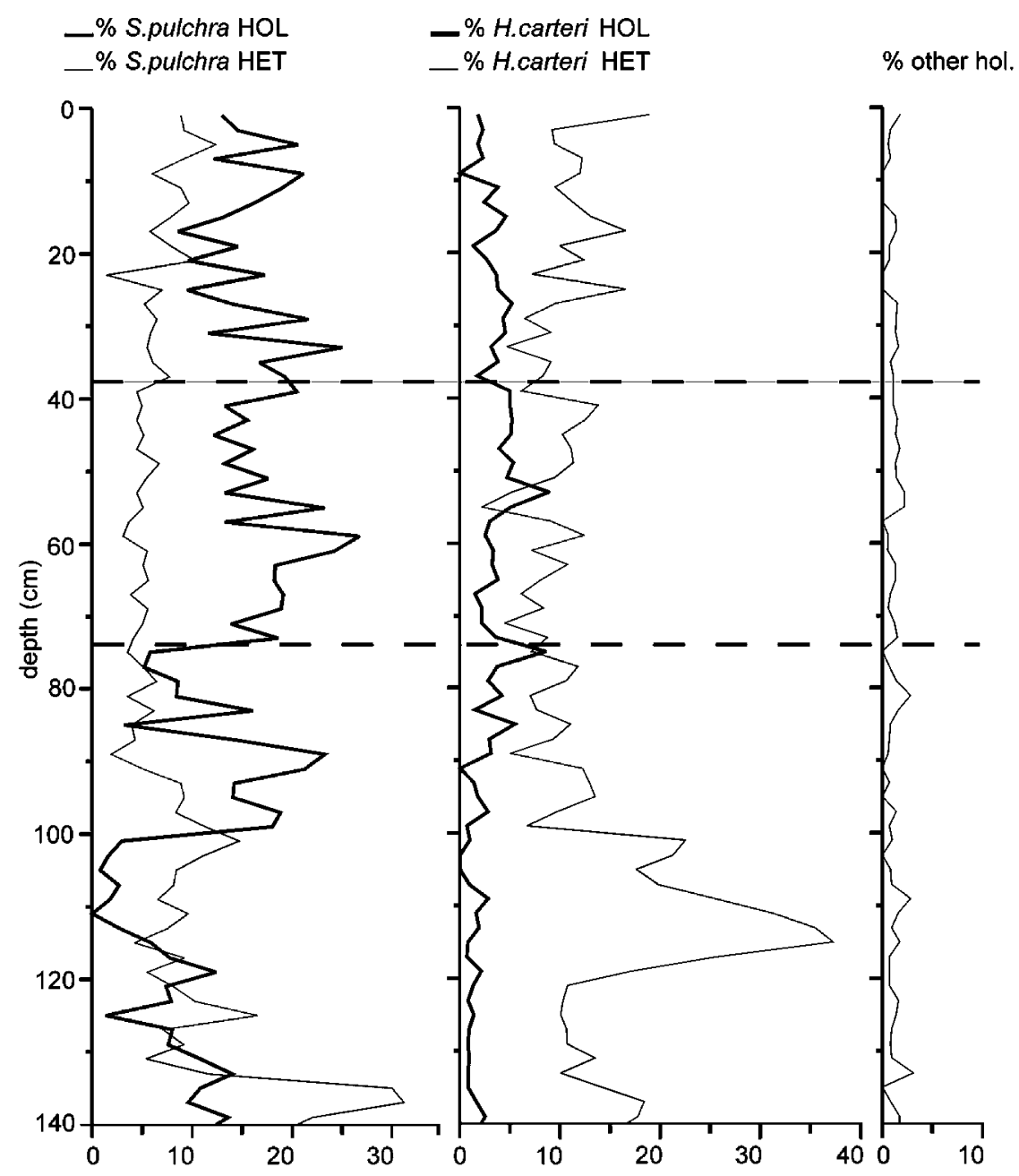

Fig. 2. Western Mediterranean. Comparison of the distribution pattern of holococcoliths and heterococcoliths produced by Syracosphaera pulchra and Helicosphaera carteri. From left to right: S. pulchra HET compared with S. pulchra HOL, H. carteri HET compared with H. carteri HOL, and "other holococcoliths" (abb., other hol.). See text for detail. The recovery depth of the core is reported.

(maximum relative abundance, $5.9 \%$ in core $\mathrm{BC} 3,37.4$ $\mathrm{cm}$ from the core top) and above the sapropel whereas they are commonly absent within it (Table 2).

\subsubsection{3. "Other holococcoliths" and unclassified calyp-} troliths. Below and above S1, "other holococcoliths" are usually $<5 \%$ but reach $5 \%$ in selected cores (Figs. $3 \mathrm{a}, 4 \mathrm{a}, \mathrm{b}$ and $5 \mathrm{a}$ ). From the bottom of $\mathrm{S} 1$ they fluctuate in abundance reaching maximum values of $10 \%$ within the visual S1 of core UM42 (Fig. 3a).

Unclassified calyptroliths roughly parallel the trend of S. pulchra HOL, being absent within the sapropel (Fig. 7b, f-g). In the shallow cores, AP01.1 and SL60, this pattern is not seen, like $S$. pulchra HOL, they occur throughout the sapropel (Fig. 7d, h).

\subsection{Holococcolith profiles in tephra}

The holococcoliths of S. pulchra suddenly decreases in abundance close to or at the tephra bases (Figs. 3b, 4a and 5a). A marked negative shift is not observed in correspondence of tephra "T" (Fig. 3a). S. pulchra HOL oblonga has low abundances close to the base of the ash layer Y-5 (Fig. 3c). H. carteri HOL and "other holococcoliths" do not show changes in abundances relative to tephras.

\subsection{Preservation of holococcoliths - scanning electron microscopy}

In samples from the visual $\mathrm{S} 1$, heavily overgrown holococcoliths were rare. Here, S. pulchra HOL cocco- 
a UM42 (1375 m)

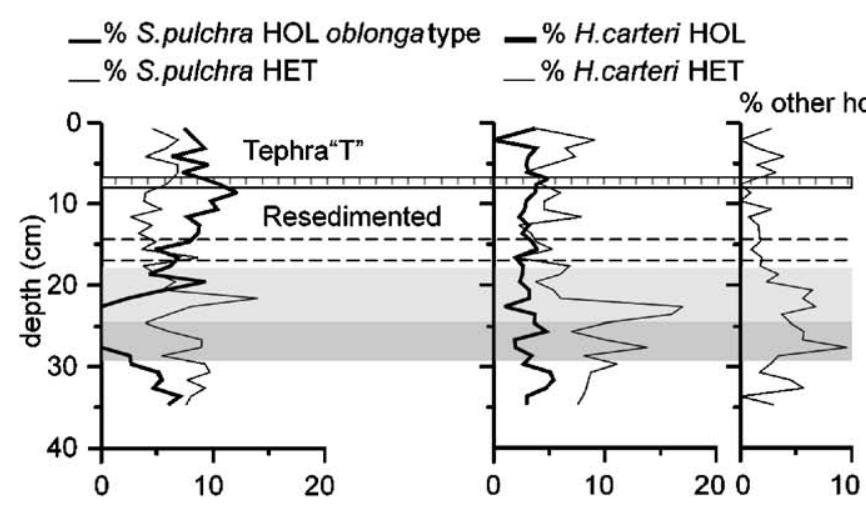

c BC3 $(2180 \mathrm{~m})$

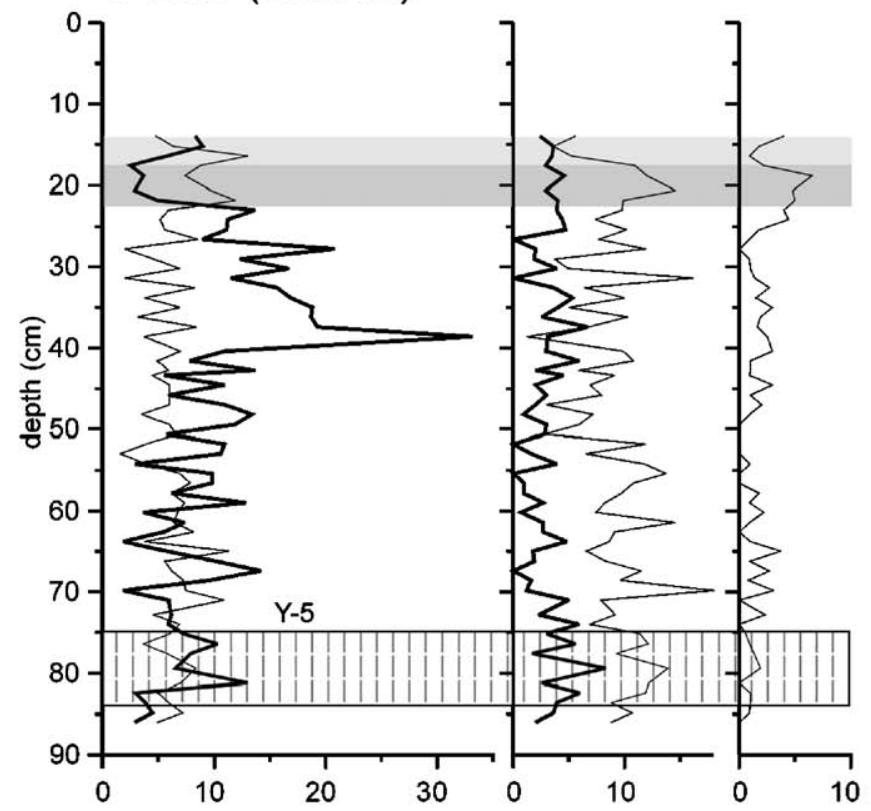

b SL114 (3390 m)

-\% S.pulchra $\mathrm{HOL} \quad-\%$ H.carteri $\mathrm{HOL}$ _\% S.pulchra HET _ $\%$ H.carteri HET \% other hol.
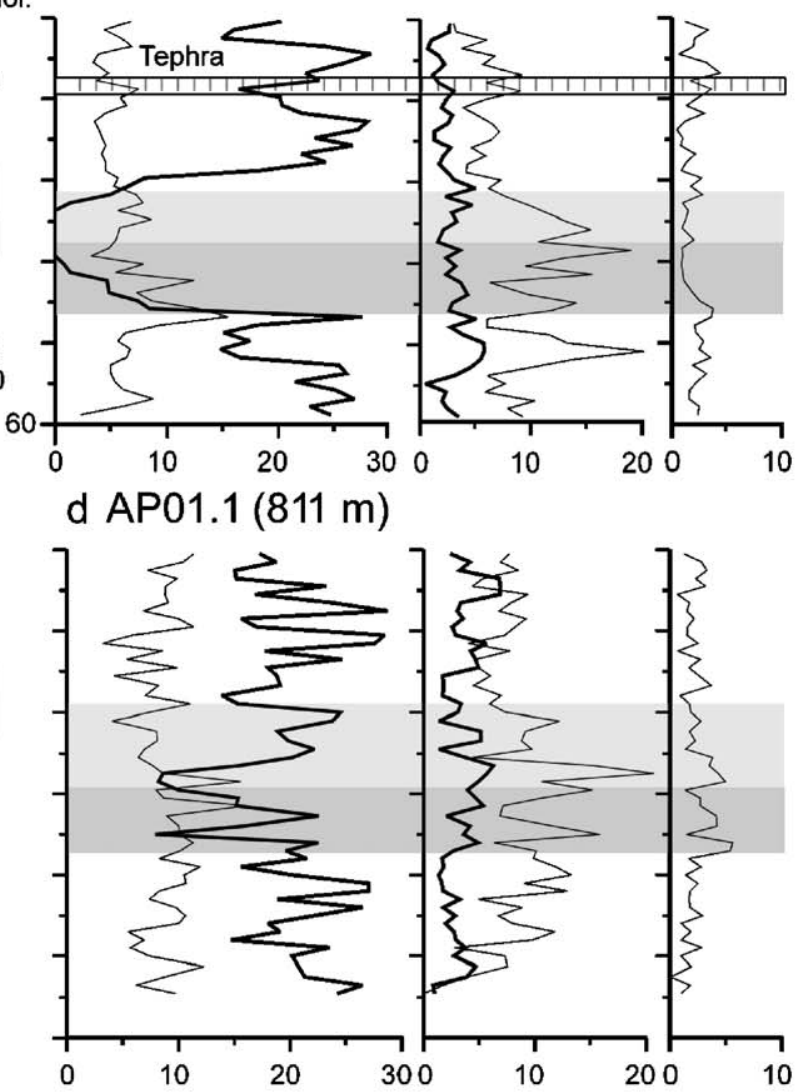

Fig. 3. Ionian Sea. Relative abundance profiles of selected coccolith associations and of "other holococcoliths". (a, c) From left to right: Syracosphaera pulchra HET compared with S. pulchra HOL oblonga type, Helicosphaera carteri HET compared with H. carteri HOL, and "other holococcoliths" (other hol.). (b, d) Taxa as in Fig. 2. Patterns of H. carteri HET and H. carteri HOL (a,c) are from Crudeli and Young (2003). The unoxidised and oxidised parts of S1 are indicated by light and dark shading. Vertical grey lines indicate tephra. For each core the recovery depth is reported.

liths displayed variable preservation including normally preserved specimens, specimens affected by mechanical breakage (Fig. 6a) partially dissolved forms (Fig. 6b), and collapsed coccoliths (Fig. 6d). Muroliths showing partial detachment of crystallites or incipient dissolution were observed (Fig. 6k, m) together with well preserved forms (Fig. 6i, j). Fragments of holococcoliths and holococcolith debris (Fig. 6b, n-p) and partially broken or dissolved heterococcoliths (Fig. 6qr) were also observed.
Normally preserved and overgrown S. pulchra HOL oblonga type and $S$. pulchra HOL pirus type coccoliths (Fig. 6c, e-f, h) and heavily overgrown unidentifiable calyptroliths (Fig. 6g) were frequent in non-sapropel samples and within the oxidised part of S1.

\subsection{Holococcoliths, morphological-structural grouping}

Based on shape and structure, the observed holococcoliths are divided into two major groups. In this work 
a BC19 $(2750 \mathrm{~m})$

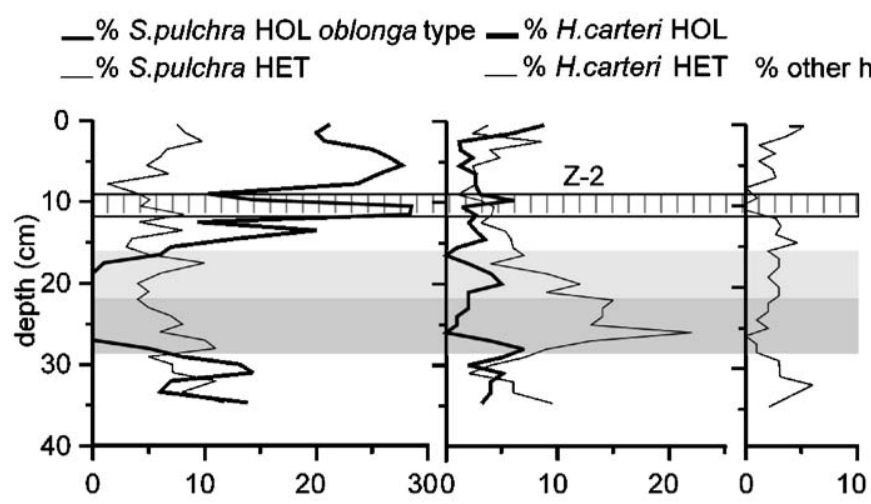

\section{b SL9 $(2302 \mathrm{~m})$}

\begin{tabular}{ll}
-\% S.pulchra HOL & $-\%$ H.carteri $\mathrm{HOL}$ \\
\hline & S.pulchra HET
\end{tabular}

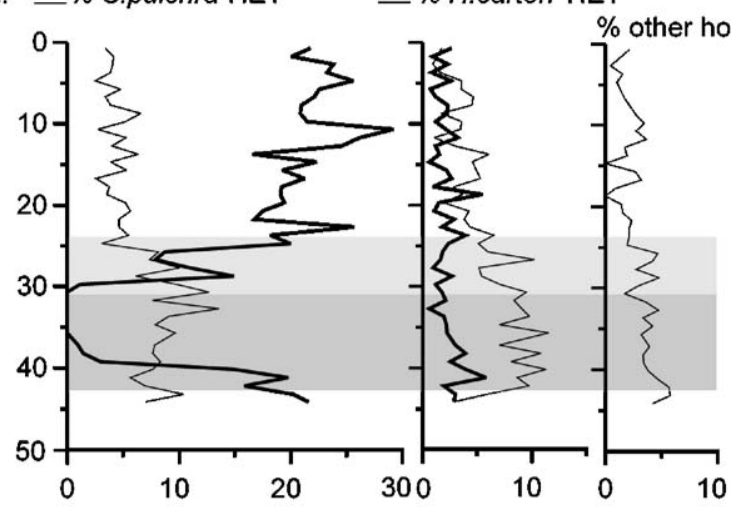

c SL29 (1578 m)

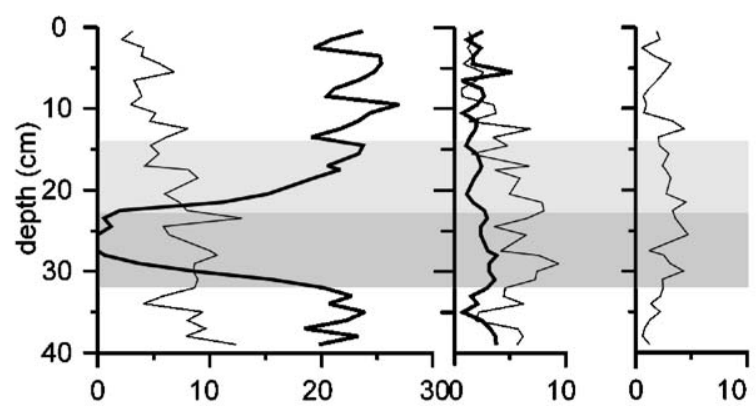

Fig. 4. Eastern Mediterranean. Relative abundance profiles of selected coccolith associations and of "other holococcoliths". (a) Taxa as in Fig. 3a and c. (b-c) Taxa as in Fig. 2. For indication of sapropel and tephra see caption to Fig. 3. For each core the recovery depth is reported.

we use syracoliths and calyptroliths (Young et al., 1997) to refer to these two groups.

The syracoliths include the $H$. carteri holococcoliths and probably most of the "other holococcoliths" group. These have compact structures formed of multiple layers of crystallites in optical continuity, thus forming large pseudo-crystals. In the case of $S$. schilleri and $S$. quadriperforatus the crystallites have vertical c-axis orientations whilst in the $H$. carteri holococcoliths the crystallites have an oblique orientation. This is important for taxonomy but is unlikely to effect diagenesis. The calyptroliths include the $S$. pulchra holococcoliths and unclassified calyptroliths. These are cavate domeshaped holococcoliths in which each crystallite is at a slightly different orientation to its neighbour and so large pseudo-crystals are not formed.

In Fig. 7 the abundances of these two holococcolith types and their sum (holococcolith spp.), in the eastern Mediterranean cores, are compared with each other and with the abundance of E. huxleyi Moderately Calcified Morphotype (EHMC). The abundance of $\mathrm{EHMC}$ is, an indicator of progressive carbonate pre- cipitation on coccoliths in these sediments (Crudeli et al., 2004).

Calyptroliths are very abundant outside S1. In general, their pattern parallels the EHMC trend. Within S1 calyptroliths are absent in those cores, where EHMC is also absent (Fig. 7a-b, e-g). In tephra layers the correlation of these forms is well visible (Fig. 7b, e). Syracoliths are less abundant than calyptroliths above and below the sapropel but they do not drastically decrease within S1.

\section{Discussion}

\subsection{Holococcoliths}

\subsubsection{Preservation potential: calyptroliths vs. syracoliths}

Holococcoliths are delicate because of their small size and the non-interlocked nature of the crystallites (e.g., Tappan, 1980). Larger, more heavily calcified holococcoliths or species with blocky morphologies are the most common types in the fossil record, whilst forms with delicate morphologies only occur in excep- 


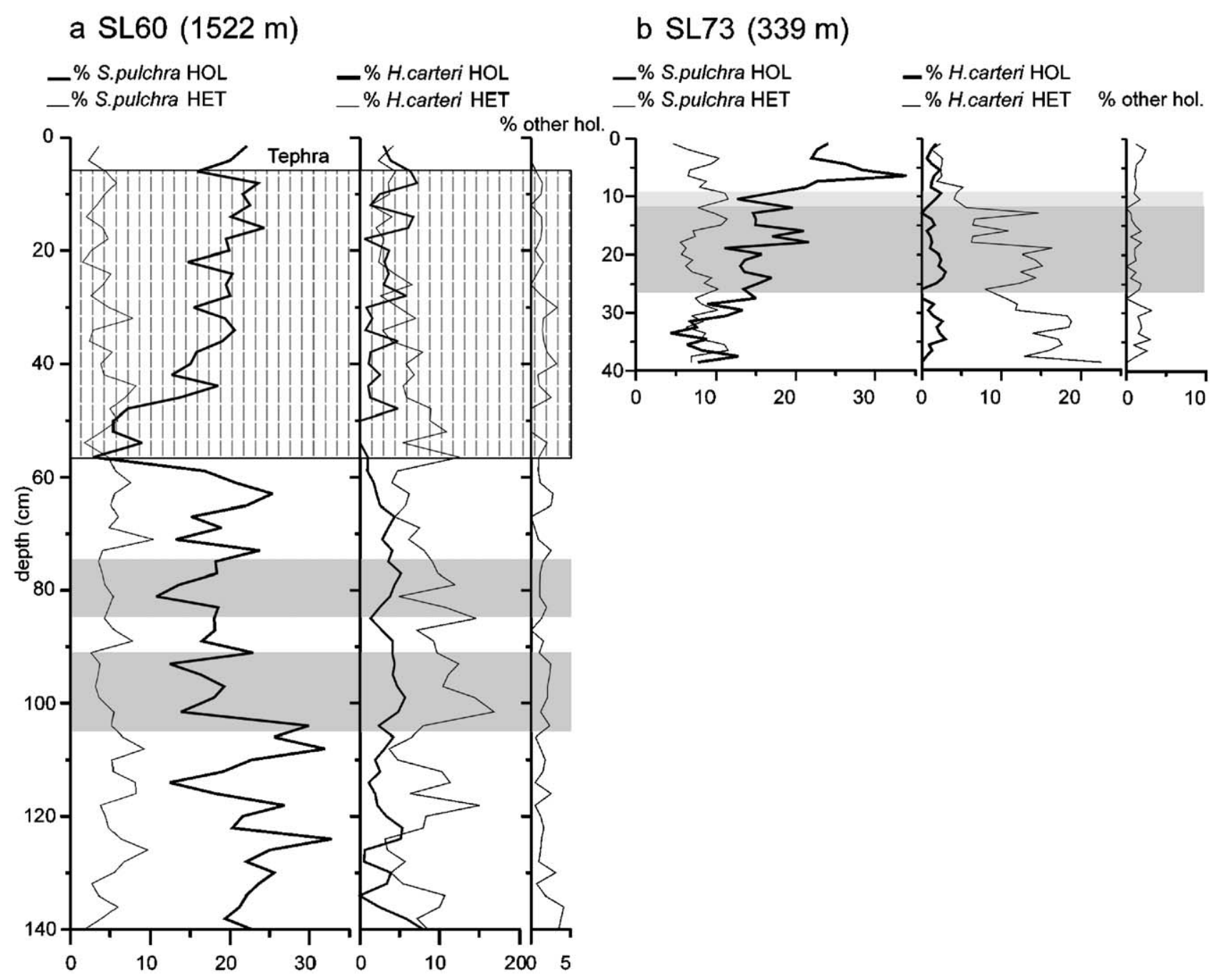

Fig. 5. Cretan (SL60) and Aegean (SL73) Sea. Relative abundance profiles of selected coccolith associations and of "other holococcoliths". Taxa as in Fig. 2. For indication of sapropel and tephra see caption to Fig. 3. For each core the recovery depth is reported. 
tionally preserved sediments (Bown, 1993, and references therein). Crystallography plays a major role in favouring overgrowth or dissolution of forms (e.g., McIntyre and McIntyre, 1971; Schneidermann, 1977).

Calyptroliths and syracoliths have different preservation potential. This is probably directly related to their distinct morphology and crystallography. Domeshaped holococcoliths are prone to overgrowth and dissolution whereas solid flat-topped holococcoliths appear to be less prone to both processes, as our qualitative SEM observations have shown. This conclusion in confirmed by the distribution of EHMC (Fig. 7).

5.1.1.1. Calyptroliths. Overgrowth of crystallites of $S$. pulchra HOL pirus type (Fig. 6e-f, h) was observed on several specimens and is probably favoured by the radial orientation of the crystallographic c-axes (perpendicular to the coccolith wall) and by the monolayered and cavate structure of the coccolith. Also these calyptroliths have a high surface-area to volume ratio which increases the possibility to be in contact with diagenetic fluids. Identifiable, overgrown S. pulchra HOL oblonga type coccoliths, are less common but are probably represented by numerous unidentifiable highly overgrown calyptroliths (Fig. 6g). Calyptroliths also appear to be prone to dissolution (Fig. 6a$b, d)$. The space between crystallites, their minute dimensions, likely favours crystallite dissolution and causes the open, dome-shaped structure to collapse.

\subsubsection{Syracoliths. H. carteri HOL, S. dalmaticus} and $S$. ponticuliferus are formed of numerous superposed layers of rhombic crystallites resulting in a solid structure (e.g., Young et al., 2003). No overgrown forms were observed by SEM although some overgrown $H$. carteri HOL coccoliths were observed by LM (Table 2). It is likely, that overgrowth of crystallites is inhibited by the compact structure. In the presence of carbonate dissolution, the solid structure probably does not favour easy transport of diagenetic fluids and prevents disintegration (Fig. 6k). S. schilleri is constituted by an open tube with internal septae with sub-vertical caxes. Despite its basically not-solid structure, it is expected to have a moderate preservation potential, as a result of its relatively large size (3-4 $\mu \mathrm{m})$. A morphologically-structurally similar holococcolith (Holodiscolithus macroporus) is almost the only holococcolith commonly preserved in Neogene sediments (PerchNielsen, 1985; Young, 1998).

\subsubsection{Preservation of a primary signal in non-sapropel sediments}

In the Western Mediterranean and Aegean Sea sediments, coccoliths are more affected by carbonate dissolution than by overgrowth (Crudeli et al., 2004) suggesting different carbonate diagenesis processes to those occurring in the eastern basin. Vazquez and Zamarreño (1993, southwestern Balearic slope) documented by SEM normal, etched and overgrown coccoliths. The type of overgrowth on coccoliths (Vazquez and Zamarreño, 1993, Fig. 8B-C; our pers. obs.) is different from that observed in the eastern basin (Crudeli et al., 2004, Plate 2) and further supports different diagenetic regime in the two basins.

Despite these likely diagenetic differences, the assemblages in the non-sapropel sediments of all the studied cores show many similarities, including, the

\footnotetext{
Fig. 6. Scanning electron micrographs of $(a-h)$ calyptrolith and $(i-m)$ syracolith holococcoliths showing different preservation within and outside sapropel S1: normal preservation, incipient dissolution, mechanical breakage and secondary calcite overgrowth, and (q-r) of other coccoliths showing breakage and dissolution. $(\mathrm{a}-\mathrm{h})$ For each image, the core and the sample depth (in $\mathrm{cm}$ ) from the top of core are reported. (i-r) All images from boxcore BC3, $21.8 \mathrm{~cm}$, visual S1. (a-p) Scale bar indicates $1 \mu \mathrm{m}$. (q-r) Scale bar indicates $2 \mu \mathrm{m}$. (a) S. pulchra HOL oblonga type, SEM micrograph of a calyptrolith in oblique view affected by mechanical breakage and partial dissolution of crystallites of the basal flange (boxcore BC3, $21.8 \mathrm{~cm}$, visual S1). (b) S. pulchra HOL pirus type, SEM micrograph of a calyptrolith in distal view showing incipient dissolution of crystallites of the basal tube. A holococcolith fragment is visible at the lower side of the image (boxcore BC3, 21.8 cm, visual S1). (c) S. pulchra HOL oblonga type, SEM micrograph of a calyptrolith in distal view with irregular overgrowth of crystallites (boxcore BC3, $0.8 \mathrm{~cm}$, outside S1). (d) S. pulchra HOL pirus type, SEM micrograph of a partially collapsed calyptrolith in oblique/distal view (boxcore BC3, 21.8 cm, visual S1). (e) SEM micrograph of $S$. pulchra HOL oblonga type in side view moderately preserved (form at the lower side) and of a calyptrolith in oblique/proximal view with overgrown crystallites of the dome-shaped structure (form in the centre) (boxcore UM42, $20.65 \mathrm{~cm}$, oxidised S1). (f) SEM micrograph of an overgrown S. pulchra HOL pirus type in oblique/side view (form in the centre) and of a normally preserved coccolith of Algirosphaera robusta in side view (form at the upper side) (boxcore UM42, $20.65 \mathrm{~cm}$, oxidised S1). (g) SEM micrograph of an unclassified overgrown calyptrolith in oblique/distal view (boxcore UM42, $22.65 \mathrm{~cm}$, oxidised S1). (h) SEM micrograph of an overgrown calyptrolith, S. pulchra HOL oblonga type?, in oblique/distal view (boxcore BC3, $0.8 \mathrm{~cm}$, outside S1). (i) H. carteri HOL solid or $H$. carteri HOL perforate, SEM micrograph of a normally preserved syracolith in oblique/proximal view. Holococcolith debris is visible at the upper-left and lower right side of the image. (j) SEM micrograph of a well preserved syracolith in distal view. (k) H. carteri HOL solid or H. carteri HOL perforate, SEM micrograph of a syracolith in oblique/proximal view affected by partial detachment of crystallites. (m) Syracolith in distal view showing partial dissolution of crystallites Holococcolith debris is visible on the upper-left side of the images. (n) Holococcolith debris. (p) Holococcolith debris. (q) Lamellar fragments of Algirosphaera robusta. (r) SEM micrograph of Emiliania huxleyi cocccoliths affected by etching and mechanical breakage.
} 
average relative abundance of $S$. pulchra HOL, $H$. carteri HOL, and "other holococcoliths", and the dominance of $S$. pulchra HOL over S. pulchra HET, and the similar abundances of $H$. carteri $\mathrm{HOL}$ and $H$. carteri HET (Figs. 2-5). This suggests only minor alteration of the primary signal.
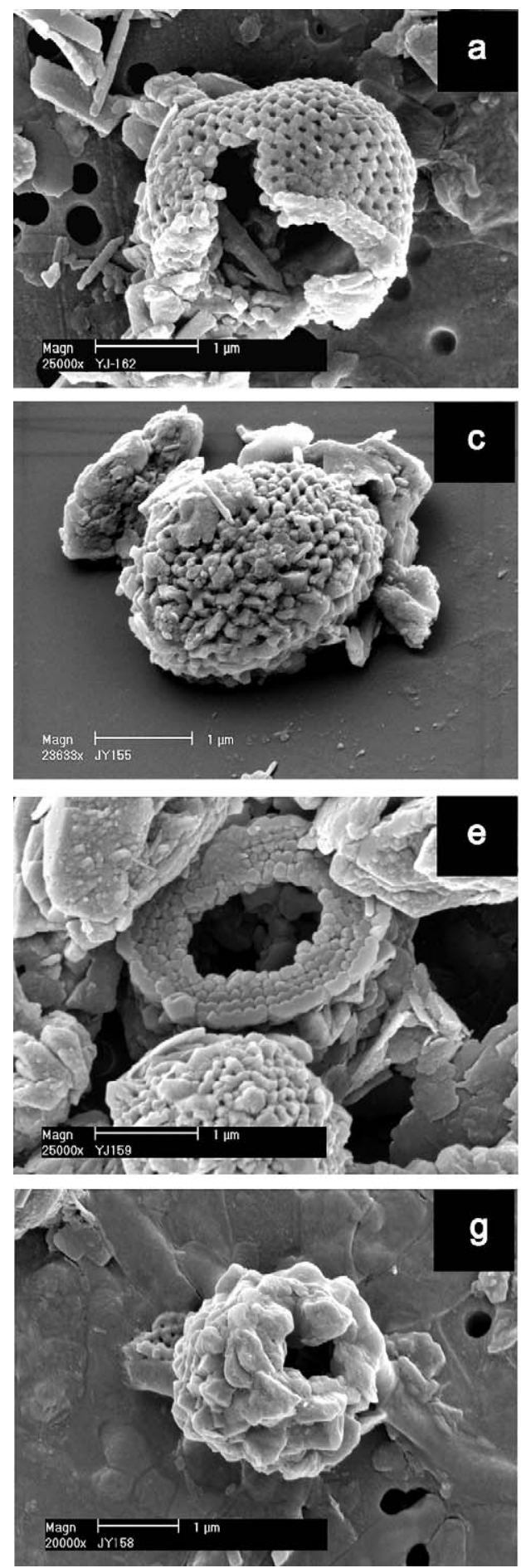

5.2. S. pulchra HET-S. pulchra HOL and H. carteri HET-H. carteri HOL-palaeoecological information from cores AP01.1 and SL60

A striking feature of the record is that S. pulchra holococcoliths are frequently more common than the
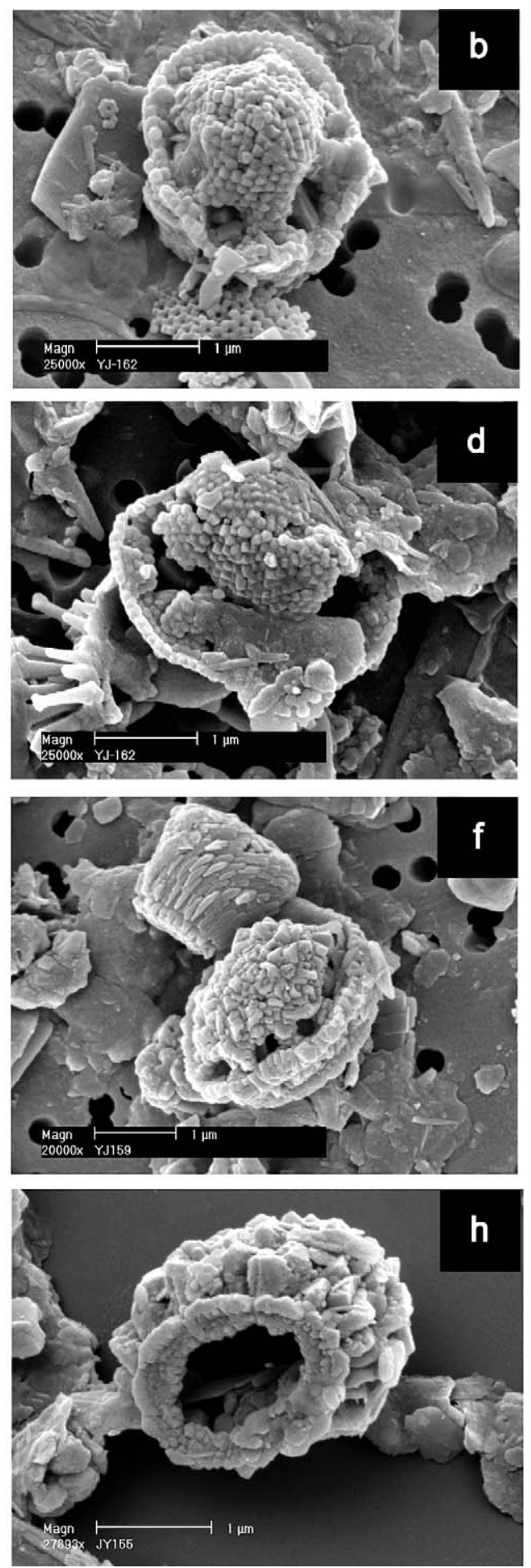
heterococcoliths (Figs. 2-5). In the plankton the two life-cycle phases are about equally common but the holococcolith phase has more coccoliths $(50-200)$ per coccosphere than the heterococcolith phase (ca. 25-40 endothecal coccoliths) so this is not an entirely unexpected result. It does, however, suggest that the holococcolith abundance has not been drastically reduced by dissolution.
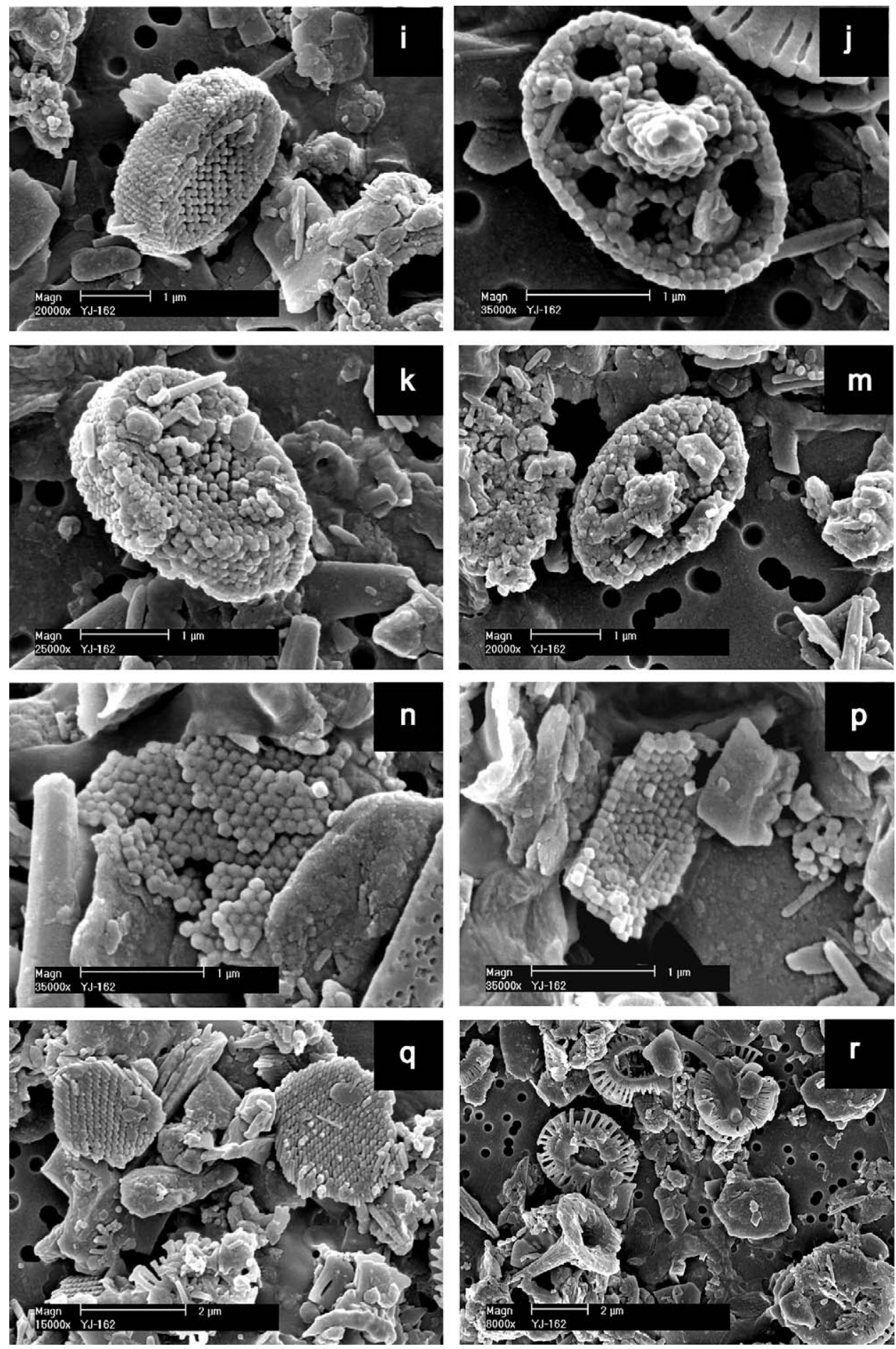

Fig. 6 (continued). 
In most cores there is a major decrease in the abundance of S. pulchra holococcoliths in the sapropel which, as discussed below, we interpret as a diagenetic effect. In the two more distant and shallower cores AP01.1 and SL60 there is virtually no evidence of this diagenetic effect and instead the holococcolith and heterococcolith stages show broadly similar distribution patterns throughout the cores (Figs. 3d and 5a). This suggests, that in these two cores there is very good preservation of the primary signal. Hence, we discuss possible palaeoecological interpretation of these cores first.

$H$. carteri HET and S. pulchra HET are both broadly characteristic of environments that are intermediate between eutrophic (dominated by Emiliania and Gephyrocapsa), and highly oligotrophic (dominated by Umbellosphaera and Discosphaera, Young, 1994). However, whilst $S$. pulchra is very widely distributed

\section{a UM42 (1375 m)}

_\% syracoliths

_\% calyptroliths
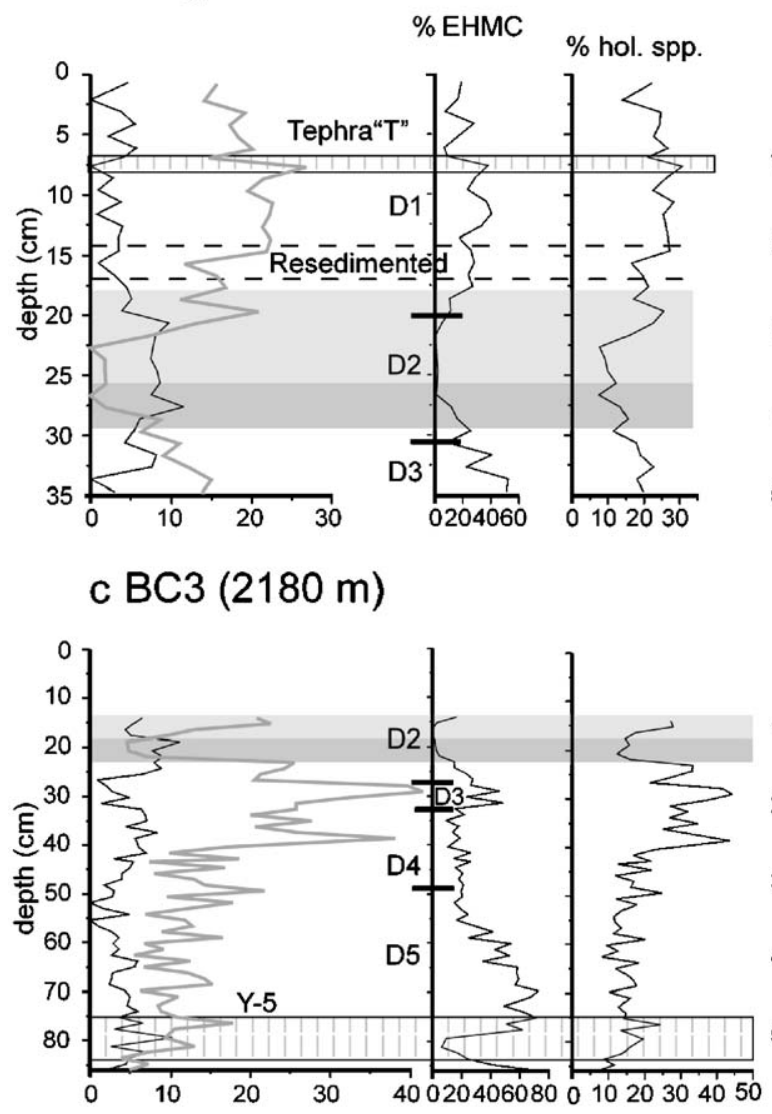

in oligo/mesotrophic waters, H. carteri has a more sporadic occurrence, seems to favour somewhat higher productivity conditions (e.g., Ziveri et al., 2004) and often has its maximum abundance in the deep chlorophyll maximum (DCM) (Cros et al., 2000). In our data $H$. carteri HET and $S$. pulchra HET show broadly inverse distribution patterns. This is particularly clear if the two life-cycle phases are summed (Fig. 8). The sapropels are intervals of increased $H$. carteri abundance vs. S. pulchra. We interpret this as reflecting higher productivity in the sapropel intervals.

Intriguingly, in both sapropel and non-sapropel sediments, peaks of abundance of S. pulchra HOL and $H$. carteri HOL often coincide with decreases of their heterococcolith phase (Figs. 3d and 5a). These patterns are not obviously related to any changes in preservation and we suspect that they record a primary ecological effect. A

\section{b SL114 (3390 m)}

\section{_\% syracoliths}

—\% calyptroliths

—\% S.pulchra HOL

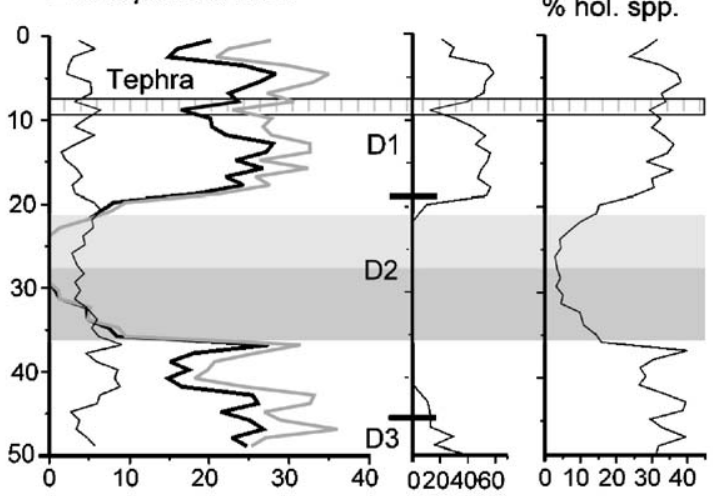

d AP01.1 (811 m)

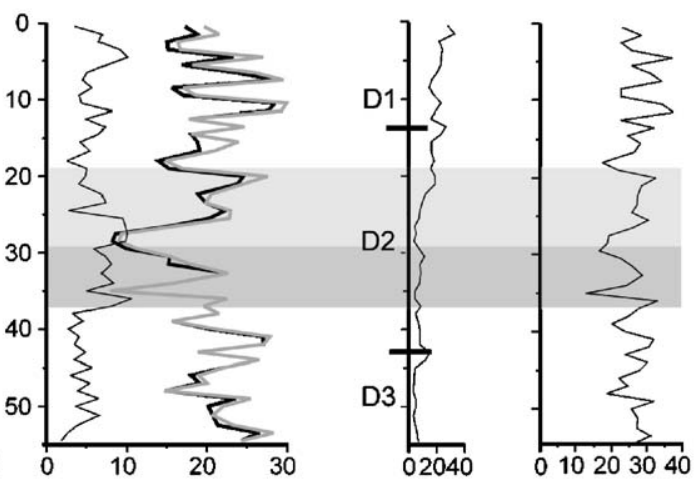

Fig. 7. Relative abundance profiles of calyptroliths and syracoliths compared with those of EHMC (Crudeli et al., 2004) and patterns of the total holococcoliths (holococcolith spp., abb. hol. spp.). The relative abundance of EHMC is the percentage in a count of 300 coccoliths. In order to visually show the abundance trend of unclassified calyptroliths, in Fig. 7b, d and f-h S. pulchra HOL and calyptroliths are compared. "Diagenetic" intervals (Crudeli et al., 2004) are indicated by shacked horizontal lines. See text for details. For indication of sapropel and tephra see caption to Fig. 3. 


\section{e BC19 $(2750 \mathrm{~m})$}

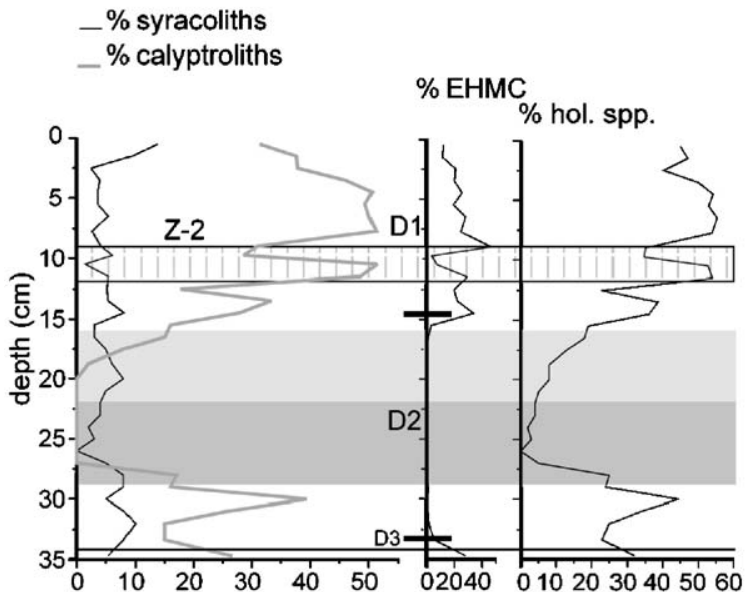

g SL29 (1587 m)

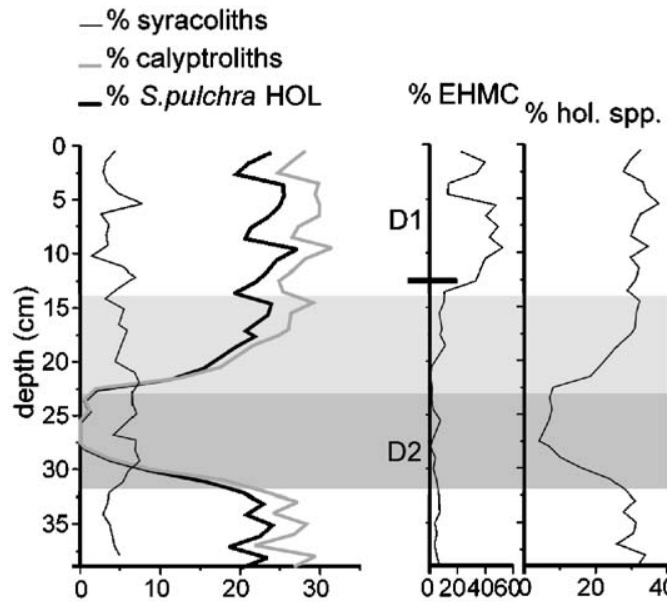

f $\operatorname{SL9}(2302 \mathrm{~m})$

_\% syracoliths

_\% calyptroliths

-\% S.pulchra HOL

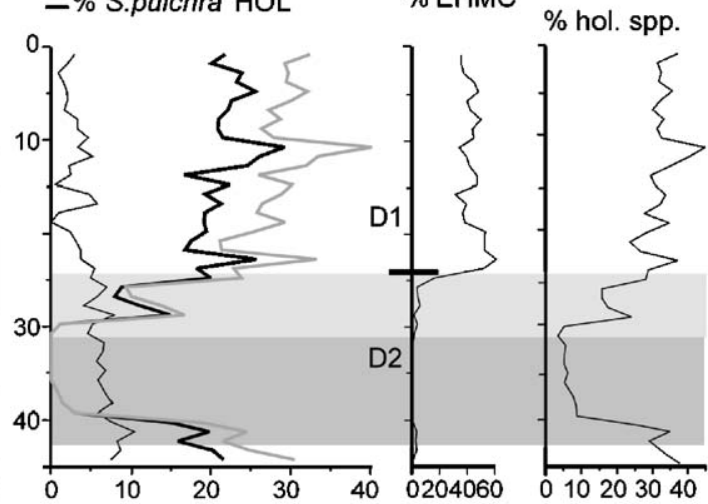

h SL60 (1522 m)

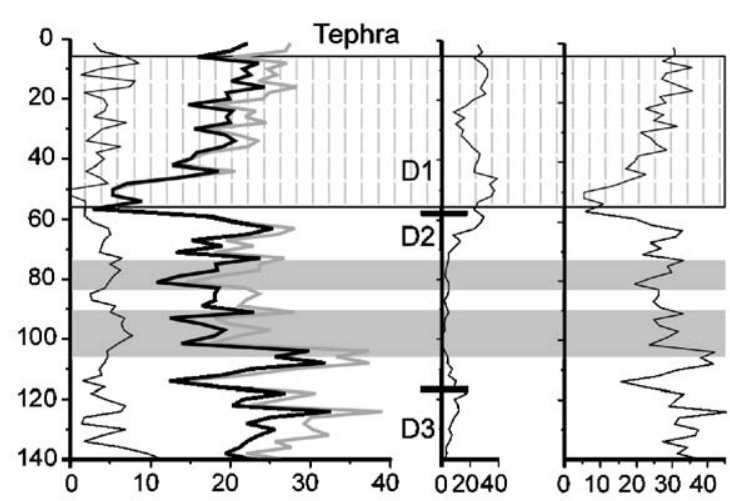

Fig. 7 (continued).

useful palaeoecological signal may be present in this data and further biological research on the significance of such changes could be of real geological value.

\subsection{Low abundance of calyptroliths within sapropel S1} in deeper Eastern Mediterranean cores, evidence of dissolution

The most striking feature of the holococcolith profiles is the major decrease in abundance of calyptroliths within the sapropels in the deeper cores (Fig. 7a-c, e-g). This decrease may be due to a primary variation, to dissolution or to a combination of the two processes. These possibilities are discussed below and by comparison with data from the shallowest cores AP01.1 and SL60.

Holococcolithophores are most abundant in oligotrophic and stratified water masses (Kleijne, 1991; Winter et al., 1994). Extensive eutrophication of surface water could have caused a reduction in holococcolith production in the central basin. However, in core B3, close to the primary source of nutrient supply into the Mediterranean (Freydier et al., 2001), the calyptroliths show only a slight decrease whereas they are absent in the more distant cores, e.g., SL114 (compare Fig. 7c and b).

It is also significant that the reduction is only seen in calyptroliths (i.e., essentially S. pulchra HOL). Different holococcolith phases almost certainly do have different ecologies, but at the current state of knowledge $H$. carteri $\mathrm{HOL}$ and $S$. pulchra HOL seem to show broadly similar ecologies and there is no obvious ecological reason why they should show very different abundance patterns in sapropels. It appears thus unlikely that the negative pattern of the calyptroliths is ecologically driven. 


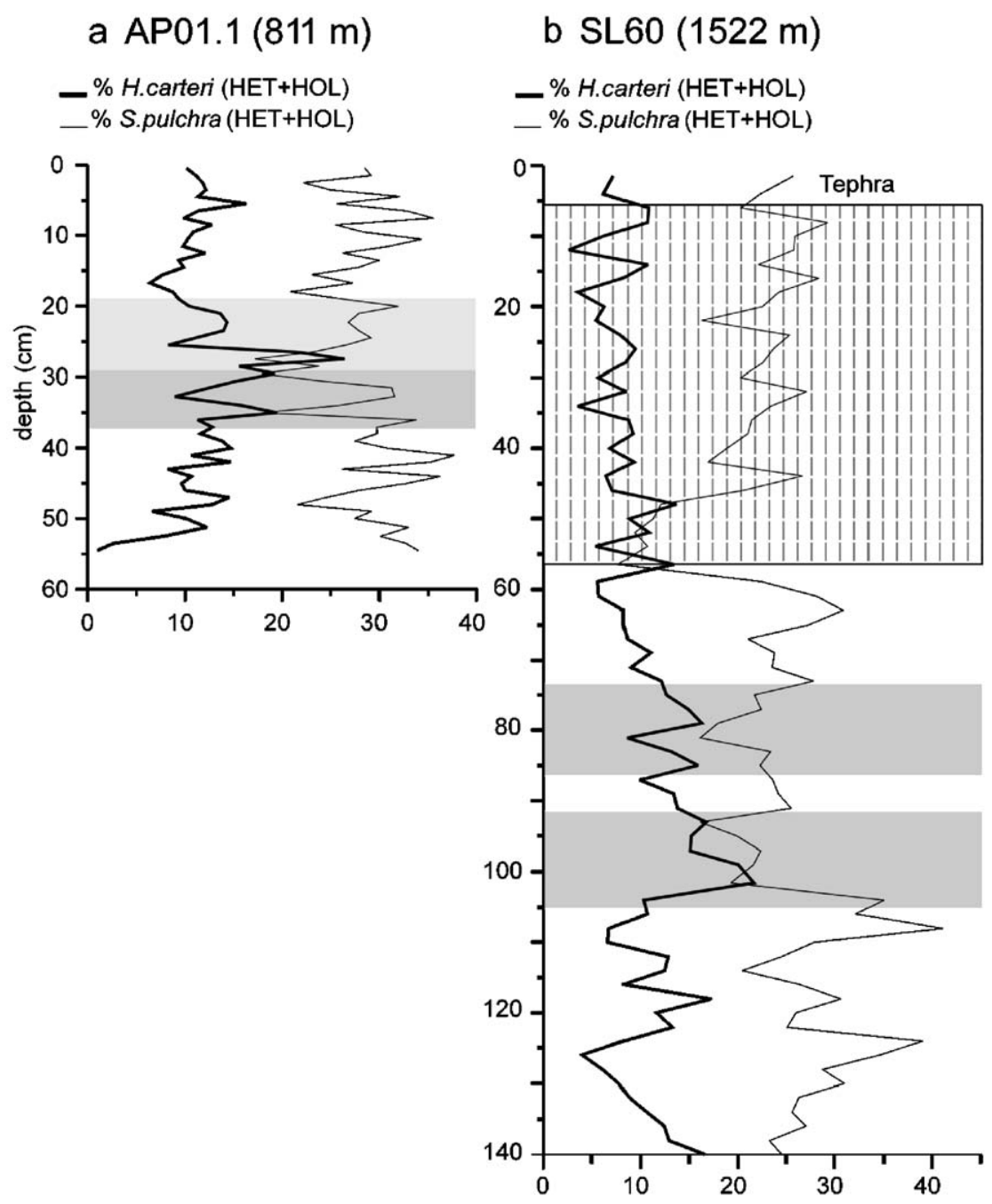

Fig. 8. Relative abundance profiles of H. carteri and S. pulchra from core AP01.1 and SL60 showing good correlation among patterns. For indication of sapropel and tephra, see caption to Fig. 3.

As previously discussed, calyptroliths are more delicate than syracoliths in relation to coccolith morphology and structure. It is thus possible that, the pattern of drastic reduction of calyptroliths within $\mathrm{S} 1$ is due to dissolution. The solid structure of the syracoliths may have favoured preservation within those intervals where calyptroliths are absent. The suggestion that the profile is a diagenetic artefact is supported by comparison of holococcolith abundance patterns (in particular those of calyptroliths) with those of $E$. huxleyi (Fig. 7). E. huxleyi Lightly Calcified Morphotype (EHLC) increases in abundance relative to the Moderately Calcified Morphotype (EHMC) in tephra layers possibly due to acidic interstitial waters that would have induced etching of E. huxleyi coccoliths (Crudeli et al., 2004). The roughly parallel decrease of calyptroliths and EHMC within tephras suggests that the pattern of the former is a dissolution artefact. Similarly, within the sapropels the decrease in calyptroliths is paralleled by a decrease in EHMC and dominance of EHLC. Particularly interesting, calyptroliths increase roughly in correspondence to the re-increase of EHMC (Fig. 7a-b, e-g) (i.e., close to the S1 top). This suggests that the re-occurrence of calyptroliths in sediments is directly related to the re-occurrence of early diagenetic carbonate precipitation at the end of S1 formation signalled by EHMC abundance. As previously discussed, calyptroliths appeared as readily overgrown forms (from SEM).

\subsubsection{Lysocline shifts or dissolution within the sediment}

The premise is that the presence of delicate holococcoliths in most S1 intervals (Fig. 7) suggests weak effects of carbonate dissolution. 
At present, Mediterranean water is calcite saturated, and delicate holococcoliths, including calyptroliths, are preserved at depth (e.g., Fig. 3). A thin, pteropod ooze surface sediment level at sites shallower than $3 \mathrm{~km}$ (de Lange et al., 1999) indicates aragonite saturation.

Pteropods are usually absent within sapropel S1, even above $3 \mathrm{~km}$ water depth. This is probably because of aragonite dissolution (Giunta et al., 2003; Principato et al., 2003) and suggests instability of the water column aragonite saturation or of the carbonate environment in the sediment. A slight variation of the calcite lysocline during sapropel formation and a successive re-deepening before the end of S1 could explain the decrease to absence of calyptroliths in the sapropel from the deeper eastern Mediterranean cores.

Alternatively, the low abundances of calytroliths may be due to subtle syndepositional variations in carbonate dissolution levels within the sapropel. It seems unlikely that the dissolution occurred during later diagenesis since the decrease in calyptroliths occurs in both the oxidised and unoxidised parts of the sapropel (e.g., Fig. 7b, e-f).

\subsection{Palaeoceanographic information}

Holococcoliths, are characteristic of oligotrophic conditions (Kleijne, 1991) so, their consistent common presence within S1 in cores SL60 and AP01.1, suggests oligotrophy of the surface water, in agreement with stratification of the basin during sapropel formation.

As discussed, the increase of $H$. carteri HET close to the S1 bottom suggests the development of a nutrientrich environment at depth. This is consistent with the DCM formation indicated by the increase in abundance and palaeofluxes of the lower-photic zone species Florisphaera profunda (e.g., Thomson et al., 2004; Principato et al., in press, respectively) and deep dwelling calcareous dinoflagellates (Meier et al., 2004). The results support a model in which nutrients from river discharge brought into the basin (Rossignol-Strick et al., 1982; Freydier et al., 2001) were trapped in the DCM supporting sub-surface productivity (Rohling and Gieskes, 1989).

\section{Conclusions}

\subsection{Preservation potential-carbonate diagenesis}

1. Of the highly diverse modern Mediterranean holococcolithophores, only a few forms are present in the sediment; S. pulchra HOL oblonga type, S. pulchra
HOL pirus type, $H$. carteri HOL solid, H. carteri HOL perforate, S. schilleri, holococcolith type F and a few other unidentified holococcoliths.

2. These holococcoliths belong to two distinct morphological-structural groups, calyptroliths and syracoliths. Calyptroliths are prone to overgrowth and dissolution whereas syracoliths are less prone to both processes. The different preservation potential is related to their morphology and microstructure.

3. In non-sapropel sediments both syracoliths and calyptroliths are preserved, but in the sapropels calyptroliths are virtually absent in deeper cores even though well-preserved syracoliths occur. This correlates with evidence from E. huxleyi morphotypes for weak carbonate dissolution in the sapropels, which may have occurred in the water column or during early diagenesis.

\subsection{Palaeoecology-palaeoceanography}

1. The inverse distribution profiles of $H$. carteri and S. pulchra is due to different ecological adaptation. The increase of $H$. carteri reflects higher productivity occurred close to the DCM during sapropel formation.

2. The variations in abundance of the life-cycle phases of the two species detected from well-preserved sediments suggests the existence of a significant palaeoecological-palaeoceanographic signal.

3. The presence of holococcoliths within S1 from the shallowest cores and their supposed original presence from other settings suggests oligotrophy of the surface water and supports the inference of concentration of nutrient in a DCM. This is further supported by the increase of $H$. carteri in the sapropel interval.

\section{Acknowledgements}

This work was supported by SAP (Sapropel and Paleoceanography) (MAS3-CT97-0137) European program. The SEM study of holococcoliths was mainly carried out at the Natural History Museum, London and supported by Sys-Resource- Access to Research Infrastructures project of the EU-IHP Program. We warmly thank C. Jones (NHM, London), A. Rizzi (Dep. of Earth Sciences, Milano), and S. Kars (Dep. of Paleoecology and Paleoclimatology, Amsterdam) for SEM assistance. We are indebted to the reviewers, A. Negri and an anonymous reviewer, whose careful reading and suggestions improved the quality of the manuscript. 


\section{References}

Billard, C., 1994. Life cycles. In: Green, J.C., Leadbeater, B.S.C. (Eds.), The Haptophyte Algae, Systematics Assoc. Spec. vol. 51. Clarendon Press, Oxford, pp. 167-186.

Bown, P.R., 1993. New holococcoliths from the Toarcian-Aalenian (Jurassic) of northern Germany. Senckenb. Lethaea 73 (2), 407-419.

Calvert, S.E., Fontugne, M.R., 2001. On the Late PleistoceneHolocene sapropel record of climatic and oceanographic variability in the Eastern Mediterranean. Paleoceanography 16 (1), $78-94$.

Castradori. D., 1992. I nannofossili calcarei come strumento per lo studio biostratigrafico e paleoceanografico del Quaternario nel Mediterraneo Orientale. Ph.D. Thesis, University of Milan, Italy.

Castradori, D., 1993. Calcareous nannofossils and the origin of Eastern Mediterranean sapropels. Paleoceanography 8 (4), $459-471$.

Cita, M.B., Vergnaud-Grazzini, C., Robert, C., Chamley, H., Ciaranfi, N., d'Onofrio, S., 1977. Paleoclimatic record of a long deep-sea core from the Eastern Mediterranean. Quat. Res. 8, 205-235.

Corselli, C., Principato, M.S., Maffioli, P., Crudeli, D., 2002. Changes in planktonic assemblages during sapropel S5 deposition: evidence from Urania Basin area, Eastern Mediterranean. Paleoceanography 17 (3). doi:10.1029/2000PA000536.

Cros, L., Kleijne, A., Zeltner, A., Billard, C., Young, J.R., 2000. New examples of holococcolith-heterococcolith combination coccospheres and their implications for coccolithophorid biology. Mar. Micropaleontol. 39, 1-34.

Cros, L., Fortuño, J-M., 2002. Atlas of Northwestern Mediterranean coccolithophores. Sci. Mar. 66 (Suppl. 1), 7-182.

Crudeli, D., Young, J.R., 2003. SEM-LM study of holococcoliths preserved in Eastern Mediterranean sediments (Holocene/Late Pleistocene). J. Nannoplankton Res. 25 (1), 29-50.

Crudeli, D., Young, J.R., Erba, E., de Lange, G.J., Henriksen, K., Kinkel, H., Slomp, C.P., Ziveri, P., 2004. Abnormal carbonate diagenesis in Holocene-Late Pleistocene sapropel-associated sediments from Eastern Mediterranean; evidence from Emiliania huxleyi coccolith morphology. In: Villa, G., Lees, J.A., Bown, P.R. (Eds.), Calcareous Nannofossil Palaeoecology and Palaeocenographic Reconstructions, Mar. Micropaleontol. vol. 52/1-4, pp. $217-240$.

de Lange, G.J., van Os, B., Pruysers, P.A., Middelburg, J.J., Castradori, D., van Santvoort, P., Müller, P.J., Eggenkamp, H., Prahl, F.G., 1994. Possible early diagenetic alteration of palaeo proxies. In: Zahn, R., Pedersen, T.F., Kaminski, M.A., Labeyrie, L. (Eds.), Carbon Cycling in the Glacial Ocean: Constraints on the Ocean's Role in Global Change, NATO ASI Series vol. I-17, pp. 225-258.

de Lange, G.J., van Santvoort, P.J.M., Langereis, C., Thomson, J., Corselli, C., Michard, A., Rossignol-Strick, M., Paterne, M., Anastasakis, G., 1999. Palaeo-environmental variations in Eastern Mediterranean sediments; a multidisciplinary approach in a prehistoric setting. Progr. Oceanogr. 44, 369-386.

Emeis, K.-C., Struck, U., Schulz, H.-M., Rosemberg, R., Bernasconi, S., Erlenkeuser, H., Sakamoto, T., Martinez-Ruiz, F., 2000. Temperature and salinity variation of Mediterranean Sea surface water over the last 16,000 years from records of planktonic stable oxygen isotopes and alkenone unsaturation ratios. Palaeogeogr. Palaeoclimatol. Palaeoecol. 158, 241-259.

Fresnel, J., 1994. A heteromorphic life cycle in two coastal coccolithophorids, Hymenomonas lacuna and Hymenomonas coronata (Prymnesiophyceae). Can. J. Bot. 72, 1455-1462.
Freydier, R., Michard, A., de Lange, G., Thomson, J., 2001. Nd isotopic compositions of Eastern Mediterranean sediments: tracers of the Nile influence during sapropel S1 formation? Mar. Geol. $177,45-62$.

Geisen, M., Billard, C., Broerse, A.T.C., Cros, L., Probert, J., Young, J.R., 2002. Life-cycle associations involving pairs of holococcolithophorid species: intraspecific variation or cryptic speciation? Eur. J. Phycol. 37, 531-550.

Geisen, M., Young, J.R., Probert, I., Sáez, A.G., Baumann, K.-H., Bollmann, J., Cros, L., Devargas, C., Medlin, L.K., Sprengel, C., 2004. Species level variation in coccolithophores. In: Thierstein, H.R., Young, J.R. (Eds.), Coccolithophores-from Molecular Processes to Global Impact. Springer, pp. 327-366.

Giunta, S., Negri, A., Morigi, C., Capotondi, L., Combourieu-Nebout, N., Emeis, K.C., Sangiorgi, F., Vigliotti, L., 2003. Coccolithophorid ecostratigraphy and multi-proxy paleoceanographic reconstruction in the Southern Adriatic Sea during the last deglacial time (Core AD91-17). Palaeogeogr. Palaeoclimatol. Palaeoecol. 190, 39-59.

Houdan, A., Billard, C., Marie, D., Not, F., Sáez, A.G., Young, J.R., Probert, I., 2004. Holococcolithophore-heterococcolithophore (Haptophyta) life cycles: flow cytometric analysis of relative ploidy levels. Syst. Biodivers. 4, 453-465.

Kleijne, A., 1991. Holococcolithophorids from the Indian Ocean, Red Sea, Mediterranean Sea and North Atlantic Ocean. Mar. Micropaleontol. 17, 1-76.

Lourens, L.J., Antonarakou, A., Hilgen, F.J., Van Hoof, A.A.M., Vergnaud-Grazzini, C., Zachariasse, W.J., 1996. Evaluation of the Plio-Pleistocene astronomical time scale. Paleoceanography 11, 391-413.

McIntyre, A., McIntyre, R., 1971. Coccolith concentrations and differential solution in oceanic sediments. In: Funnel, B., Riedel, W.B. (Eds.), The Micropaleontology of Oceans. Cambridge University Press, pp. 253-261.

Meier, K.J.S., Zonneveld, K.A.F., Kasten, S., Willems, H., 2004. Different nutrient sources forcing increased productivity during Eastern Mediterranean S1 sapropel formation as reflected by calcareous dinoflagellate cysts. Paleoceanography 19. doi:10.1029/ 2003PA000895.

Mercone, D., Thomson, J., Croudace, I.W., Siani, G., Paterne, M., Troelstra, S., 2000. Duration of S1, the most recent sapropel in the eastern Mediterranean Sea, as indicated by AMS radiocarbon and geochemical evidence. Paleoceanography 15, $336-347$.

Negri, A., Capotondi, L., Keller, J., 1999. Calcareous nannofossils, planktonic foraminifera and oxygen isotopes in the Late Quaternary sapropels of the Ionian Sea. Mar. Geol. 157, 89-103.

Noël, M.-H., Kawachi, M., Inouye, I., 2004. Induced dimorphic life cycle of a Coccolithophorid, Calyptrosphaera sphaeroidea (Prymnesiophyceae, Haptophyta). J. Phycol. 40, 112-129.

Parke, M., Adams, I., 1960. The motile (Crystallolithus hyalinus Gaarder and Markali) and non-motile phases in the live-history of Coccolithus pelagicus (Wallich) Schiller. J. Mar. Biol. Assoc. UK 39, 263-274.

Perch-Nielsen, K., 1985. Cenozoic calcareous nannofossils. In: Bolli, H.M., Saunders, J.B., Perch-Nielsen, K. (Eds.), Plankton Stratigraphy. Cambridge Univ. Press, Cambridge, pp. 427-554.

Principato, M.S., 2003. Late Pleistocene-Holocene planktonic foraminifera from the Eastern Mediterranean Sea: towards a highresolution planktonic foraminiferal assemblage zonation for the Late Quaternary of the Mediterranean. Riv. Ital. Paleontol. Stratigr. 109 (1), 111-124. 
Principato, M.S., Giunta, S., Corselli, C., Negri, A., 2003. Late Pleistocene-Holocene planktonic assemblages in three box-cores from the Mediterranean Ridge area (west-southwest of Crete): palaeoecological and palaeoceanographic reconstruction of sapropel S1 interval. Palaeogeogr. Palaeoclimatol. Palaeoecol. 190, 61-77.

Principato, M.S., Crudeli, D., Ziveri, P., Slomp, C.P., Corselli, C., Erba, E., de Lange, G.J., in press. Phyto- and zooplankton paleofluxes during the deposition of sapropel S1 (eastern Mediterranean): biogenic carbonate preservation and paleoecological implications. Palaeogeogr. Palaeoclimatol. Palaeoecol.

Rohling, E.J., 1994. Review and new aspects concerning the formation of Eastern Mediterranean sapropels. Mar. Geol. 122, 1-28.

Rohling, E.J., Gieskes, W.W.C., 1989. Late Quaternary changes in Mediterranean Intermediate water density and formation rate. Paleoceanography 4, 531-545.

Rowson, J.D., Leadbeater, B.S.C., Green, J.C., 1986. Calcium carbonate deposition in the motile (Crystallolithus) phase of Coccolithus pelagicus (Prymnesiophyceae). Br. Phycol. J. 21, 359-370.

Rossignol-Strick, M., 1985. Mediterranean Quaternary sapropels, an immediate response of the African monsoon to variation of insolation. Palaeogeogr. Palaeoclimatol. Palaeoecol. 49, 237-263.

Rossignol-Strick, M., Nesteroff, W., Olive, P., Vergnaud-Grazzini, C., 1982. After the deluge - Mediterranean stagnation and sapropel formation. Nature 295, 105-110.

Roth, P.H., Coulbourn, W.T., 1982. Floral and dissolution patterns of coccoliths in surface sediments of the North Pacific. Mar. Micropaleontol. 7, 1-52.

Sáez, A.G., Probert, I., Geisen, M., Quinn, P., Young, J.R., Medlin, L.K., 2003. Pseudo-cryptic speciation in coccolithophores. Proc. Nat. Acad. Sci. 100 (12), 7163-7168.

Saugestad, A.H., Heimdal, B.R., 2002. Light microscope studies on coccolithophorids from the Western Mediterranean Sea, with notes on combination cells of Daktylethra pirus and Syracosphaera pulchra. Plant Biosyst. 136 (1), 3-28.

Sbaffi, L., Wezel, F.C., Kallel, N., Paterne, M., Cacho, I., Ziveri, P., Shackleton, N., 2001. Response of the pelagic environment to palaeoclimatic changes in the central Mediterranean Sea during the Late Quaternary. Mar. Geol. 178, 39-62.

Schneidermann, N., 1977. Selective dissolution of recent coccoliths in the Atlantic Ocean. In: Ramsay, A.T.S. (Ed.), Oceanic Micropaleontology vol. 2. Academic Press, London, pp. 1009-1053.

Steinmetz, J.C., 1991. Calcareous nannoplankton biocoenosis: sediment trap studies in the Equatorial Atlantic, Central Pacific, and Panama Basin. Ocean Biocoenosis Series No. 1. Woods Hole Oceanogr. Inst., 85.

Tappan, H., 1980. Haptophyta, coccolithophores, and other calcareous nannoplankton. The Paleobiology of Plant Protists. Freeman W.H. and Company, San Francisco, pp. 678-803.
Thierstein, H.R., Young, J.R., 2004. Coccolithophores-from molecular processes to global impact. Springer.

Thomson, J., Crudeli, D., de Lange, G.J., Slomp, C.P., Erba, E., Corselli, C., Calvert, S.E., 2004. Florisphaera profunda and the origin and diagenesis of carbonate phases in Eastern Mediterranean sapropel units. Paleoceanography 19 (3). doi:10.1029/ 2003PA000976.

Triantaphyllou, M.V., Dimiza, M.D., 2003. Verification of the Algirosphaera robusta-Sphaerocalyptra quadridentata (coccolithophores) life-cycle association. J. Micropalaeontol. 22, 107-111.

van Santvoort, P.J.M., de Lange, G.J., Thomson, J., Cussen, H., Wilson, T.R.S., Krom, M.D, Strohle, K., 1996. Active post-depositional oxidation of the most recent sapropel (S1) in sediments of the Eastern Mediterranean Sea. Geochim. Cosmochim. Acta 60, 4007-4024.

Vazquez, A., Zamarreño, I., 1993. Late Quaternary hemipelagic carbonate oozes on the southwestern Balearic slope (Western Mediterranean). Mar. Geol. 112, 71-87.

Winter, A., Jordan, R.W., Roth, P.H., 1994. Biogeography of living coccolithophores in ocean water. In: Winter, A., Siesser, W.G. (Eds.), Coccolithophores. Cambridge Univ. Press, Cambridge, pp. $171-177$.

Young, J.R., 1994. Functions of coccoliths. In: Winter, A., Siesser, W.G. (Eds.), Coccolithophores. Cambridge Univ. Press, Cambridge, pp. $63-82$.

Young, J.R., 1998. Neogene nannofossils. In: Bown, P.R. (Ed.), Calcareous Nannofossil Biostratigraphy, British Micropalaeontology Society Series. Kluwer Academic Publisher, pp. 225-265.

Young, J.R., Bergen, J.A., Bown, P.R., Burnett, J.A., Fiorentino, A., Jordan, R.W., Kleijne, A., van Niel, B.E., Romein, A.J.T., von Salis, K., 1997. Guidelines for coccolith and calcareous nannofossil terminology. Palaeontology 40, 875-912.

Young, J.R., Davis, S.A., Bown, P.R., Mann, S., 1999. Coccolith ultrastructure and biomineralisation. J. Struct. Biol. 126, 195-215.

Young, J.R., Geisen, M., Cros, L., Kleijne, A., Sprengel, C., Probert, J., Østergaard, J., 2003. A guide to extant coccolithophore taxonomy. J. Nannoplankton Res. Spec. Issue 1, 1-125.

Young, J.R., Geisen, M., Probert, J., 2005. A review of selected aspects of coccolithophore biology with implications for palaeobiodiversity estimation. Micropaleontology 51 (4).

Ziveri, P., Broerse, A.T.C., van Hinte, J.E., Westbroek, P., Honjo, S., 2000. The fate of coccoliths at $48^{\circ} \mathrm{N} 21^{\circ} \mathrm{W}$, Northeastern Atlantic. Deep-Sea Res. 47, 1853-1875.

Ziveri, P., Baumann, K.-.H., Böckel, B., Bollmann, J., Young, J.R., 2004. Biogeography of selected Holocene coccoliths in the Atlantic Ocean. In: Thierstein, H.R., Young, J.R. (Eds.), Coccolithophores-from Molecular Processes to Global Impact. Sprinter, pp. 403-428. 